\title{
Nuclear shape coexistence in Po isotopes: An interacting boson model study
}

\author{
J. E. García-Ramos ${ }^{1}$ and K. Heyde ${ }^{2}$ \\ ${ }^{1}$ Departamento de Física Aplicada, Universidad de Huelva, 21071 Huelva, Spain \\ ${ }^{2}$ Department of Physics and Astronomy, Ghent University, Proeftuinstraat, 86 B-9000 Gent, Belgium \\ (Received 22 May 2015; revised manuscript received 17 July 2015; published 11 September 2015)
}

\begin{abstract}
Background: The lead region, $\mathrm{Po}, \mathrm{Pb}, \mathrm{Hg}$, and $\mathrm{Pt}$, shows up the presence of coexisting structures having different deformation and corresponding to different particle-hole configurations in the shell-model language.

Purpose: We intend to study the importance of configuration mixing in the understanding of the nuclear structure of even-even Po isotopes, where the shape coexistence phenomena are not clear enough.

Method: We study in detail a long chain of polonium isotopes, ${ }^{190-208} \mathrm{Po}$, using the interacting boson model with configuration mixing (IBM-CM). We fix the parameters of the Hamiltonians through a least-squares fit to the known energies and absolute $B(E 2)$ transition rates of states up to $3 \mathrm{MeV}$.

Results: We obtained the IBM-CM Hamiltonians and we calculate excitation energies, $B(E 2)$ 's, electric quadrupole moments, nuclear radii and isotopic shifts, quadrupole shape invariants, wave functions, and deformations.

Conclusions: We obtain a good agreement with the experimental data for all the studied observables and we conclude that shape coexistence phenomenon is hidden in Po isotopes, very much as in the case of the Pt isotopes.
\end{abstract}

DOI: 10.1103/PhysRevC.92.034309

PACS number(s): 21.10.-k, 21.60.Fw, 27.80.+w

\section{INTRODUCTION}

Shape coexistence has been observed by now all through the nuclear mass region, encompassing light nuclei $\left({ }^{16} \mathrm{O}\right.$ region) [1], proceeding up to the region of very heavy nuclei in the $\mathrm{Pb}$ region $[2,3]$, and has been reviewed in a number of papers over a period spanning about 3 decades [4-6]. Recent advances in experimental methods to explore nuclei, removed far from the region of $\beta$-stable nuclei, have opened up possibilities to explore the appearance and behavior of shape coexistence in series of isotopes and isotones [7]. A wealth of new data on energy systematics, but, more importantly, on observables such as in-beam spectroscopy and lifetime data [8,9], Coulomb excitation using inverse kinematics [10], direct reactions on unstable nuclei [11], radioactive-decay modes at the limits of nuclear stability [12], and breakup reactions [13], have been at the basis of recognizing the rather universal appearance of shape coexisting phenomena [6]. Moreover, measurement of the essential ground-state properties, such as masses [14], charge radii [15], and nuclear moments [16], as well as the possibilities to study monopole $E 0$ transitions in nuclei [17,18], helped to complete the data basis in such a way as to confront theoretical modeling of nuclear structure properties in greater detail than before.

From a theoretical side, present-day methods starting from the nuclear shell model or approaching the atomic nucleus using mean-field methods have resulted in developments of new algorithms as well as making use of the increased computing possibilities (see Refs. [19-21] and references therein). The present status has evolved in a situation where the conditions for shape coexistence to occur are becoming understood. It looks like a balance between two opposing nuclear force components, i.e., on one side the stabilizing effect caused by the presence of closed shells (the monopole part), aiming at stabilizing the nucleus into a spherical shape, and on the other side the low-multipole (mainly quadrupole) components redistributing protons and neutrons into a deformed shape, is at the origin of the appearance of shape coexistence in a given mass region. Recently, large-scale shell-model studies, using diagonalization in a very large many-open-shell basis in various mass regions [22] or making use of an advanced Monte Carlo shell-model approach [21], have been carried out. Besides, the concept to start from deformed average potentials and calculating the total energy curves as a function of deformation has been explored, in particular, for nuclei in the $\mathrm{Pb}$ region [23-25]. However, recent studies [26], using a microscopic approach to determine the optimal mean fields, even going beyond bringing in the nuclear dynamics, have given quantitative results - using both Skyrme forces [26-29] and Gogny forces [30-34], as well as making use of a relativistic mean-field approach [35-40]-that are indicative of the above mechanism. Moreover, attempts have been made and are still being improved to extract a Bohr Hamiltonian $[41,42]$ starting from a microscopic basis [43-45].

From a microscopic shell-model point of view, the hope to treat on equal footing the large open neutron shell from $N=126$ down to and beyond the midshell $N=104$ region, jointly with the valence protons in the $\mathrm{Pt}, \mathrm{Hg}, \mathrm{Po}$, and $\mathrm{Rn}$ nuclei, even including proton multiparticle multihole (mp-nh) excitations across the $Z=82$ shell closure, is beyond present computational possibilities. The truncation of the model space, however, by concentrating on nucleon pair modes (mainly $0^{+}$and $2^{+}$ coupled pairs, to be treated as bosons within the interacting boson approximation (IBM) [46]), has made calculations feasible, even including pair excitations across the $Z=82$ shell closure $[47,48]$ in the $\mathrm{Pb}$ region in a transparent way. More in particular, the $\mathrm{Pb}$ nuclei have been extensively studied, giving rise to bands with varying collectivity depending on the nature of the excitations treated in the model space [49-54]. More recently, detailed studies of the Pt nuclei have been carried out [55-61], as well as for the Hg nuclei [62-64], in an attempt to describe the large amount of low-lying states and their $E 2$ decay properties, explicitly including particle-hole excitations 
across the $Z=82$ shell closure. A novel mapping procedure to determine an algebraic IBM Hamiltonian has been proposed by Nomura et al. $[65,66]$. In contrast to the standard shell-model to boson-model mapping method $[67,68]$, it has been shown that an IBM Hamiltonian can be determined, mapping a self-consistent mean-field total energy surface $E(\beta, \gamma)$ (over the full $\beta-\gamma$ plane) onto the corresponding IBM mean-field energy. Very recently, this method was extended to include intruder mp-nh configurations, with a detailed coverage of the $\mathrm{Pt}, \mathrm{Pb}$, and $\mathrm{Hg}$ isotopes [69-72].

Recently, a lot of new experimental results have become available in the $\mathrm{Pb}$ region, encompassing, besides the $\mathrm{Pb}$ isotopes, the nearby $\mathrm{Hg}, \mathrm{Pt}$, and Po isotopes (see Sec. II for references on the $\mathrm{Po}$ nuclei). In both the $\mathrm{Pb}$ and the $\mathrm{Hg}$ isotopes, there is overwhelming information [excitation energies, $B(E 2)$ values, isotopic shifts, $\alpha$-hindrance factors, etc.] by now that highlights the presence of shape coexistence, which is associated with the presence of prolate, oblate, and spherical bands in the case of the $\mathrm{Pb}$ nuclei and with the presence of a prolate deformed band together with a less deformed oblate structure, forming the yrast band in the case of $\mathrm{Hg}$ [8]. Whereas the intruder bands are easily singled out for the $\mathrm{Pb}$ and $\mathrm{Hg}$ nuclei in which the excitation energies display the characteristic parabolic pattern with minimal excitation energy around the $N=104$ neutron midshell nucleus, this structure is not immediate in both the Pt and the Po nuclei. Therefore, in the present paper, we carry out an extensive study of the Po nuclei within the context of the IBM, including $2 \mathrm{p}-2 \mathrm{~h}$ excitations across the $Z=82$ proton closed shell, thereby extending the regular IBM model space, containing $N$ bosons, with the intruder $N+2$ IBM model space, also taking into account the interaction between both subspaces, which is called the interacting boson model with configuration mixing (IBM-CM) approach.

The paper is organized as follows. In Sec. II, we describe the experimental situation in the Po isotopes, whereas in Sec. III, we present the various theoretical approaches that have been used in the literature to study the Po nuclei. In Sec. IV, we succinctly present the IBM-CM formalism as well as the fitting methodology used; here, we also discuss the main outcome of the calculations on energy spectra and electric quadrupole properties $[B(E 2)$ values, quadrupole moments] and its comparison with the available experimental data. In Sec. V, we discuss the results on $\alpha$-hindrance factors as well as on the isotopic shifts. Sec. VI is devoted to the description of nuclear deformation properties of Po nuclei as derived from the IBM-CM mean-field energy, from the study of the quadrupole shape invariants and from the study of the kinematic moments of inertia, characterizing the yrast band structure of Po nuclei. Moreover, we compare the present results with the nearby $\mathrm{Pb}$, $\mathrm{Hg}$, and Pt isotopes. Finally, in Sec. VII, both the main conclusions as well as an outlook for further studies are presented.

\section{EXPERIMENTAL DATA: SITUATION IN THE Po NUCLEI}

The even-even Po nuclei span a large region of isotopes, starting with the lightest presently known ${ }^{190}$ Po nucleus $(N=$ 106), passing through the end of the shell, ${ }^{210}$ Po, all the way up to $N=134$ at ${ }^{218}$ Po.

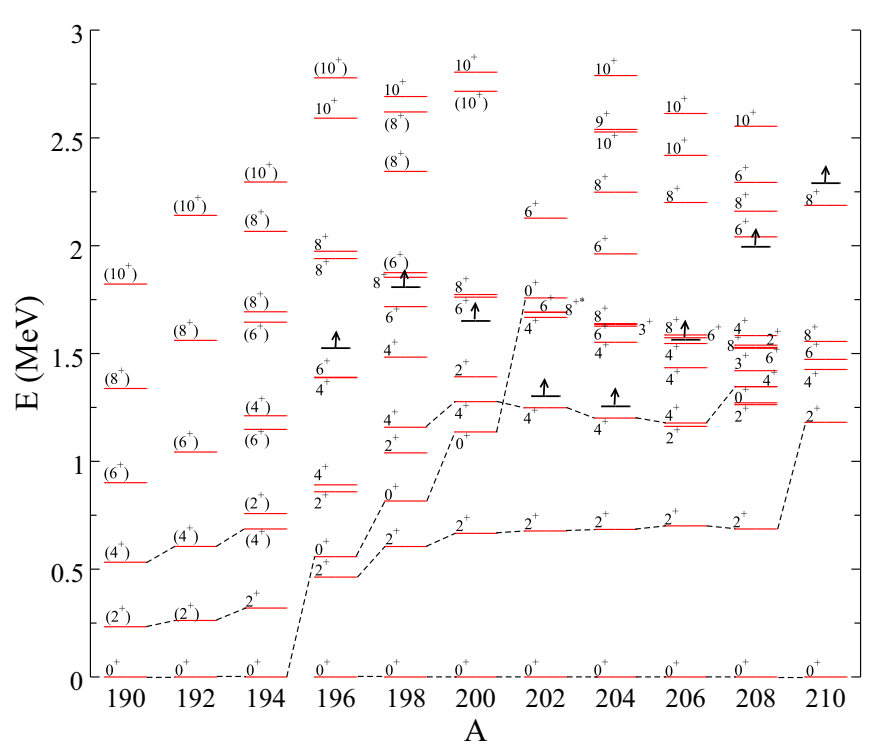

FIG. 1. (Color online) Experimental energy-level systematics for the Po isotopes. Only levels up to $E_{x} \sim 3.0 \mathrm{MeV}$ are shown. The symbol $\uparrow$ indicates the beginning of the region where extra levels of low spin, indicated with spin, parity assignments between brackets in the Nuclear Data Sheet references, start appearing. The symbol * for the $8^{+}$level for mass $A=202$ indicates that the energy is within an interval $\leqslant 40 \mathrm{keV}$ above the $6^{+}$level. Dashed lines connect states that are supposed to have a similar structure.

Many experimental complementary methods have been used to disentangle the properties over such a large interval. These nuclei are extensively covered in the Nuclear Data Sheet reviews for $A=190$ [73], $A=192$ [74], $A=194$ [75], $A=196$ [76], $A=198$ [77], $A=200$ [78], $A=202$ [79], $A=204$ [80], $A=206$ [81], $A=208$ [82], $A=210$ [83], and span the region we concentrate on in the present paper.

The experimental information (up to the end of the 1990s), down to $A=192(N=108)$, was obtained mainly using early fusion-evaporation reactions, followed by in-beam $\gamma$-ray spectroscopy, with, in particular, information on energy spectra systematics for $A=192,194$ [84-86], $A=194$ [87], $A=$ 196, 198 [88], $A=196,198$ [89], $A=198$ [90], and $A=198$, 200 [91]. This particular part of the mass region has also been analyzed using $\alpha$ - and $\beta$-decay studies of mass-separated Rn and At nuclei, resulting in information about excited $0^{+}$states in the isotopes with $A=196-202$ [92] and in energy spectra of the isotopes $A=200,202$ [93], as well as making use of $\alpha$-decay studies from the Rn nuclei for $A=198$ [94]. $\alpha$-decay studies, moving from the Po nuclei into the $\mathrm{Pb}$ nuclei, have been a major fingerprint, in particular, in view of the shape coexisting structure in the daughter $\mathrm{Pb}$ nuclei. There exists an extensive set of results that has been accumulated during a period of about 2 decades [95-109].

It was observed that mass $A=194$ (see Fig. 1) indicated a break in the energy scale observed for the heavier masses, where the energy of the $2_{1}^{+}$level is typically of the order of $\sim 600-650 \mathrm{keV}$, going down to an energy of about $\sim 300 \mathrm{keV}$, therefore, dropping by a factor two. More recently, experimental studies spanning the 1999-2009 
period have been performed at the Department of Physics at the University of Jyväskylä using the recoil-decay-tagging (RDT) technique, studying prompt and delayed $\gamma$ rays down to mass $A=190$ [110,111]. Moreover, lifetimes could be derived making use of the RDT technique in recoil distance Doppler-shift experiments for masses $A=194$ [112,113] and $A=196$ [114]. The experimental situation for the Po isotones down to $A=190$ has been summarized by Julin et al. [8]. Very recently, Coulomb excitation experiments have been performed using postaccelerated ${ }^{196,198,200,202} \mathrm{Po}$ beams at REX-ISOLDE, resulting in an important set of specific reduced $E 2$ matrix elements, connecting the $0_{1}^{+}$ ground state with the excited $2_{1,2}^{+}$states (as well as for other combinations) [115]. Charge radii have been measured for both the odd- $A A=191-211$ Po nuclei [116,117] and the even-even $A=192-210,216$, and 218 Po nuclei [118], extending the ground-state information in a major way.

\section{THEORETICAL APPROACHES: THE EVOLVING SITUATION IN THE Po NUCLEI}

The experimental situation, as discussed before, hints for a particular change in the structure moving down in mass number $A$. The energy scale, which starts at the $N=126$ closed neutron shell, set by the seniority scheme [119-121], with a first excited $2^{+}$state at $1181 \mathrm{keV}$, quickly sets in a rather constant value of the $2^{+}$energy at $\sim 650 \mathrm{keV}$. However, over the span of a few mass units one notices (i) a quick drop of the first excited $0^{+}$from mass $A=202(1758 \mathrm{keV})$, down to mass $A=196(558 \mathrm{keV})$, and (ii) a considerable drop in the $2_{1}^{+}$energy, starting at mass $A=196$ (at $463 \mathrm{keV}$ ) and setting into a new energy scale (of $\sim 250 \mathrm{keV}$ ) for the lower masses (known only down to $A=190$ at present).

In the early works of Refs. [87,89] a description using a particle-core coupling model approach was proposed, resulting from coupling the two proton particles with a vibrational core system. With the experimental information on masses $A=$ 196 and later $A=194$, a systematic study was carried out in Refs. [122,123] for the mass region $A=210$ down to $A=$ 194. Here it was pointed out that the quick drop of, in particular, the first excited $0_{2}^{+}$state, arose because of the need to use an enhanced particle-core coupling strength. This point was later stressed by Oros et al. [124], who showed that a consistent set of coupling strengths was unable to describe the observed properties, in particular, below mass $A=200$.

Calculations using a deformed average field [125] indicated that in studying the energy surfaces, while at mass $A=196$, a soft spherical result was obtained, at mass $A=192$, an oblate minimum appeared, becoming the lowest minimum at a value of $\epsilon=-0.2$ and an almost degenerate situation at $A=190$ with both oblate and prolate minima with both $\epsilon$ values of $\sim 0.2$. Energy surface calculations, covering the $\beta-\gamma$ plane were carried out using a deformed Woods-Saxon potential, resulting in the presence of three minima in the mass region $A=190$ to $A=186$ [124]. The above results were confirmed later by Smirnova et al. [126], this time using a self-consistent Hartree-Fock-Bogoliubov approach using the SLy4 Skyrme force [26], indicating rather wide minima in $A=196-194$, a lowest oblate minimum at $A=192$ and
$A=190$, and a turning into prolate at $A=188$. Triggered by the new data on masses $A=196$ and $A=194$, more detailed beyond-mean-field studies were carried out [112114], highlighting a detailed comparison on energy spectra and $E 2$ properties, indicating the interplay of an oblate and a more spherical structure (vibrational). In their analysis, the authors point towards a rather pure intruder character of the whole yrast band, including the ground state. In our analysis (see Sec. IV E), we come to the conclusion that the ground state exhibits a rather mixed character instead of being of pure intruder nature. More recently, a full study of even-even nuclei in the $\mathrm{Pb}$ region [127], showing specific results for the Po nuclei, is presented. It is interesting to point out that high-spin isomers have been studied in the Po, too, making use of a deformed Woods-Saxon potential, showing the effects of deformation on the specific excitation energy [128,129].

Most of the mean-field studies point towards the existence of rather complex energy surfaces with the presence of several minima, although in many cases separated by small barriers. Anyhow, a common denominator is the presence of a regular configuration, slightly deformed (oblate or $\gamma$ unstable) or spherical, coexisting with an intruder configuration of prolate nature, corresponding with a larger deformation as compared with the regular configuration. The lack of full calculations in the $\beta-\gamma$ plane makes difficult to formulate a more precise conclusion about the particular shape of the coexisting minima.

Within the framework of the shell model and allowing for both the full neutron open shell, covering $N=126$ to $N=82$, and the proton mp-mh excitations across the $Z=82$ closed shell, the calculations are unfeasible nowadays. Therefore, a truncated approach can be used starting from the standard IBM allowing for the presence of extra pairs. This method, called IBM-CM was proposed by Duval and Barrett [47,48] and has been used in the study of shape coexistence in various mass regions. Some early studies were carried out within the idea of a possible symmetry to be used within an extended version of the IBM, including particle-hole pairs (namely intruder $I$ spin $[130,131])$, with specific applications to the Po nuclei (Refs. [124,132]) in which the coupling between U(5) and SU(3) symmetries were explored and compared to the thenexisting data (mass $A=200$ down to $A=192$ ). Besides, a different symmetry, i.e., $F$-spin symmetry [46], was proposed to relate energy spectra in nuclei with different numbers of protons, $N_{\pi}$, and neutrons pairs, $N_{\nu}$, outside of the nearest closed shells, however keeping the sum $F=\left(N_{\pi}+N_{v}\right) / 2$ constant. An application to the $\mathrm{Pb}$ region was carried out by Barrett et al. [133]. The data obtained recently [134-140] from detailed studies of the $\mathrm{Hg}$ nuclei on energy spectra and electric quadrupole properties [ $B(E 2)$ values, $Q$ moments], however, do indicate that the more simple idea of $I$ spin is not holding so well.

\section{THE INTERACTING BOSON MODEL WITH CONFIGURATION MIXING FORMALISM}

\section{A. The formalism}

The IBM-CM is an extension of the original IBM, making it possible to treat simultaneously several boson configurations that correspond to different particle-hole ( $\mathrm{p}-\mathrm{h})$ shell-model 
excitations [48]. In our case, the model space includes the regular proton $2 p$ configurations and a number of valence neutrons outside of the $Z=82, N=126$ closed shells (corresponding to the standard IBM treatment for the Po even-even nuclei), as well as the proton $2 \mathrm{~h}-4 \mathrm{p}$ configurations and the same number of valence neutrons corresponding to a $[N] \oplus[N+2]$ boson space ( $N$ being the number of active protons, counting both proton holes and particles, plus the number of valence neutrons outside the $Z=82, N=126$ closed shells, divided by 2 as the boson number). Consequently, the Hamiltonian for two-configuration mixing can be written as

$$
\hat{H}=\hat{P}_{N}^{\dagger} \hat{H}_{\text {ecqf }}^{N} \hat{P}_{N}+\hat{P}_{N+2}^{\dagger}\left(\hat{H}_{\text {ecqf }}^{N+2}+\Delta^{N+2}\right) \hat{P}_{N+2}+\hat{V}_{\text {mix }}^{N, N+2},
$$

where $\hat{P}_{N}$ and $\hat{P}_{N+2}$ are projection operators onto the $[N]$ and $[N+2]$ boson spaces, respectively, $\hat{V}_{\operatorname{mix}}^{N, N+2}$ describes the mixing between the $[N]$ and the $[N+2]$ boson subspaces, and

$$
\hat{H}_{\text {ecqf }}^{i}=\varepsilon_{i} \hat{n}_{d}+\kappa_{i}^{\prime} \hat{L} \cdot \hat{L}+\kappa_{i} \hat{Q}\left(\chi_{i}\right) \cdot \hat{Q}\left(\chi_{i}\right),
$$

is a restricted IBM Hamiltonian called extended consistent- $Q$ (ECQF) Hamiltonian [141,142] with $i=N, N+2, \hat{n}_{d}$ the $d$ boson number operator,

$$
\hat{L}_{\mu}=\left[d^{\dagger} \times \tilde{d}\right]_{\mu}^{(1)}
$$

the angular momentum operator, and

$$
\hat{Q}_{\mu}\left(\chi_{i}\right)=\left[s^{\dagger} \times \tilde{d}+d^{\dagger} \times s\right]_{\mu}^{(2)}+\chi_{i}\left[d^{\dagger} \times \tilde{d}\right]_{\mu}^{(2)}
$$

the quadrupole operator. This approach has been proven to be a good approximation in several recent papers on $\mathrm{Pt}[57,58]$ and $\mathrm{Hg}$ isotopes [64].

The parameter $\Delta^{N+2}$ can be associated with the energy needed to excite two proton particles across the $Z=82$ shell gap, giving rise to $2 \mathrm{p}-2 \mathrm{~h}$ excitations, corrected for the pairing interaction gain and including monopole effects $[49,143]$. The operator $\hat{V}_{\text {mix }}^{N, N+2}$ describes the mixing between the $N$ and the $N+2$ configurations and is defined as

$$
\begin{aligned}
\hat{V}_{\mathrm{mix}}^{N, N+2}= & w_{0}^{N, N+2}\left(s^{\dagger} \times s^{\dagger}+s \times s\right) \\
& +w_{2}^{N, N+2}\left(d^{\dagger} \times d^{\dagger}+\tilde{d} \times \tilde{d}\right)^{(0)} .
\end{aligned}
$$

The $E 2$ transition operator for two-configuration mixing is subsequently defined as

$$
\hat{T}(E 2)_{\mu}=\sum_{i=N, N+2} e_{i} \hat{P}_{i}^{\dagger} \hat{Q}_{\mu}\left(\chi_{i}\right) \hat{P}_{i}
$$

where the $e_{i}(i=N, N+2)$ are the effective boson charges and $\hat{Q}_{\mu}\left(\chi_{i}\right)$ the quadrupole operator defined in Eq. (4).

In Sec. IV B we present the methods used to determine the parameters appearing in the IBM-CM Hamiltonian as well as in the $\hat{T}(E 2)$ operator.

The wave function, within the IBM-CM, can be described as

$$
\begin{aligned}
\Psi(k, J M)= & \sum_{i} a_{i}^{k}(J ; N) \psi\left((s d)_{i}^{N} ; J M\right) \\
& +\sum_{j} b_{j}^{k}(J ; N+2) \psi\left((s d)_{j}^{N+2} ; J M\right),
\end{aligned}
$$

where $k, i$, and $j$ are rank numbers. The weight of the wave function contained within the $[N]$-boson subspace, can then be defined as the sum of the squared amplitudes $w^{k}(J, N) \equiv$ $\sum_{i}\left|a_{i}^{k}(J ; N)\right|^{2}$. Likewise, one obtains the content in the $[N+$ 2]-boson subspace.

\section{B. The fitting procedure: Energy spectra and absolute $B(E 2)$ reduced transition probabilities}

Here we present the way in which the parameters of the Hamiltonian (1), (2), (4), and (5) and the effective charges in the $\hat{T}(E 2)$ transition operator (6) have been determined. We study the range ${ }^{190} \mathrm{Po}$ to ${ }^{208} \mathrm{Po}$, thereby covering almost the whole second half of the neutron shell $N=82-126$.

In the fitting procedure carried out here, we try to obtain the best possible agreement with the experimental data, including both the excitation energies and the $B(E 2)$ reduced transition probabilities. Using the expression of the IBM-CM Hamiltonian, as given in Eq. (1), and of the E2 operator, as given in Eq. (6), in the most general case 13 parameters show up. We impose as a constraint to obtain parameters that change smoothly in passing from isotope to isotope. Note also that we constrained $\chi_{N}=0$ and $\kappa_{N+2}^{\prime}=0$. We have explored in detail the validity of this assumption and we have found very little improvement in the value of $\chi^{2}$ [see Eq. (8)] when releasing those parameters. However, we have kept the value that describes the energy needed to create an extra particle-hole pair (two extra bosons) constant, i.e., $\Delta^{N+2}=2400 \mathrm{keV}$ and have also applied the constraint of keeping the mixing strengths constant too, i.e., $w_{0}^{N, N+2}=w_{2}^{N, N+2}=30 \mathrm{keV}$ for all the Po isotopes. We also have to determine for each isotope the effective charges of the $E 2$ operator. This finally leads to eight parameters to be varied in each nucleus.

To determine the value of $\Delta^{N+2}$, we considered as a reference the already known values for $\mathrm{Pt}$ and $\mathrm{Hg}$, which are 2800 and $3480 \mathrm{keV}$, respectively. On one hand, we expect to get a value closer to $\mathrm{Pt}$ than to $\mathrm{Hg}$ because the intruder states for Po are supposed to be very close to the regular ones and even become the ground state around the midshell. That constrains our value to be not much higher than $2800 \mathrm{keV}$. On the other hand, we know that the heavier isotopes, near the closed shell, should have an energy gap between the regular and the intruder states that is equal, at maximum, to $\Delta^{N+2}$ (see Fig. 2 on correlation energy) and, moreover, experimentally the intruder states should appear above $2000-3000 \mathrm{keV}$.

To determine the value of the mixing strengths, we considered that the corresponding value for the Pt nuclei was fixed to $50 \mathrm{keV}$ [57], while for the $\mathrm{Pb}$ it was fixed to a smaller strength of $18 \mathrm{keV}$ [52,53], and for $\mathrm{Hg}$ it was fixed to $20 \mathrm{keV}$. We performed a set of exploratory calculations between 20 and $30 \mathrm{keV}$ and found that the best overall agreement corresponds to $w_{0}^{N, N+2}=w_{2}^{N, N+2}=30 \mathrm{keV}$, although the agreement is quite similar over the whole range.

The $\chi^{2}$ test is used in the fitting procedure to extract the optimal solution. The $\chi^{2}$ function is defined in the standard way as

$$
\chi^{2}=\frac{1}{N_{\text {data }}-N_{\text {par }}} \sum_{i=1}^{N_{\text {data }}} \frac{\left[X_{i}(\text { data })-X_{i}(\text { IBM })\right]^{2}}{\sigma_{i}^{2}},
$$




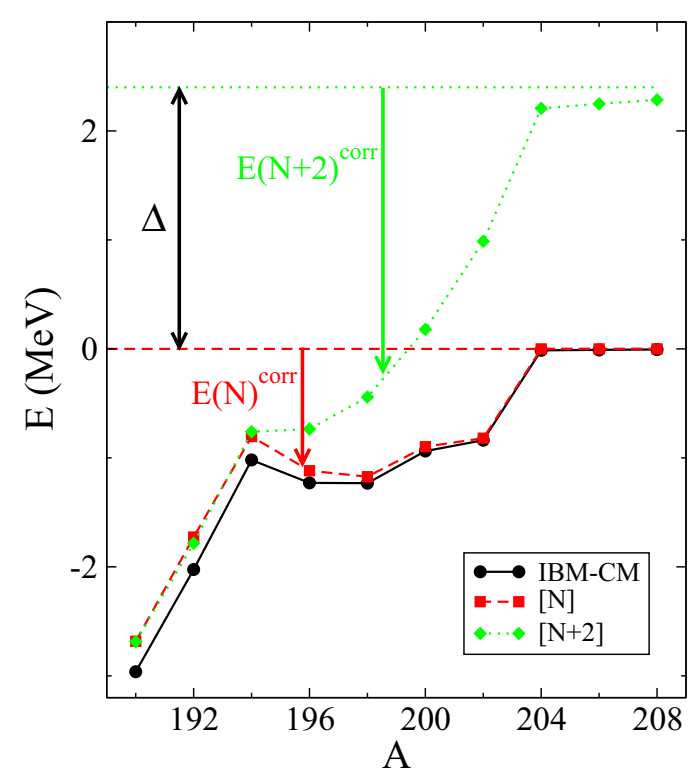

FIG. 2. (Color online) Absolute energy of the lowest unperturbed regular and intruder $0_{1}^{+}$states for ${ }^{190-208}$ Po. The arrows correspond to the correlation energies in the $N$ and $N+2$ subspaces (see also the text for a more detailed discussion).

where $N_{\text {data }}$ is the number of experimental data, $N_{\text {par }}$ is the number of parameters used in the IBM fit, $X_{i}$ (data) describes the experimental excitation energy of a given excited state [or an experimental $B(E 2)$ value], $X_{i}(\mathrm{IBM})$ denotes the corresponding calculated IBM-CM value, and $\sigma_{i}$ is an error (theoretical) assigned to each $X_{i}$ (data) point. We minimize the $\chi^{2}$ function for each isotope separately using the package MINUIT [144], which makes it possible to minimize any multivariable function.

In some of the lighter Po isotopes, owing to the small number of experimental data, the values of some of the free parameters could not be fixed unambiguously using the above fitting procedure. Moreover, for the heavier isotopes ( $A$ > 202), that part of the Hamiltonian corresponding to the intruder states is fixed such as to guarantee that those states appear well above the regular ones, that is, above $2 \mathrm{MeV}$. In some cases, owing to the lack of experimental data, the effective charges could not be determined.

As input values, we have used the excitation energies of the levels presented in Table I. In this table we also give the

TABLE I. Energy levels, characterized by $J_{i}^{\pi}$, included in the energy fit, if known, and the assigned $\sigma$ values in $\mathrm{keV}$.

\begin{tabular}{lc}
\hline \hline Error $(\mathrm{keV})$ & States \\
\hline$\sigma=0.1$ & $2_{1}^{+}$ \\
$\sigma=1$ & $4_{1}^{+}, 0_{2}^{+}, 2_{2}^{+}$ \\
$\sigma=10$ & $2_{3}^{+}, 3_{1}^{+}, 4_{2}^{+}, 6_{1}^{+}, 8_{1}^{+}$ \\
$\sigma=100$ & $6_{2}^{+}$ \\
\hline \hline
\end{tabular}

TABLE II. Hamiltonian and $\hat{T}(E 2)$ parameters resulting from the present study. All quantities have the dimension of energy (given in units of $\mathrm{keV}$ ), except $\chi_{N+2}$, which is dimensionless, and $e_{N}$ and

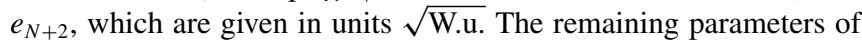
the Hamiltonian, i.e., $\chi_{N}$ and $\kappa_{N+2}^{\prime}$, are equal to zero, except for $\Delta^{N+2}=2400 \mathrm{keV}$ and $w_{0}^{N, N+2}=w_{2}^{N, N+2}=30 \mathrm{keV}$.

\begin{tabular}{lccrccccc}
\hline \hline Nucleus & \multicolumn{1}{c}{$\varepsilon_{N}$} & \multicolumn{1}{c}{$\kappa_{N}$} & \multicolumn{1}{c}{$\kappa_{N}^{\prime}$} & $\varepsilon_{N+2}$ & $\kappa_{N+2}$ & $\chi_{N+2}$ & $e_{N}$ & $e_{N+2}$ \\
\hline${ }^{190} \mathrm{Po}$ & 712.6 & -29.41 & 6.34 & 285.43 & -28.56 & 0.22 & $2.88^{\mathrm{a}}$ & $1.86^{\mathrm{a}}$ \\
${ }^{192} \mathrm{Po}$ & 731.8 & -24.20 & 0.43 & 402.67 & -30.09 & 0.21 & $2.88^{\mathrm{a}}$ & $1.86^{\mathrm{a}}$ \\
${ }^{194} \mathrm{Po}$ & 800.3 & -15.12 & -16.00 & 518.23 & -28.92 & 0.41 & 2.88 & 1.86 \\
${ }^{196} \mathrm{Po}$ & 845.0 & -22.76 & -10.96 & 373.16 & -31.02 & 0.15 & 1.86 & 1.86 \\
${ }^{198} \mathrm{Po}$ & 982.3 & -28.00 & -25.74 & 854.82 & -34.51 & 1.33 & 2.05 & 1.10 \\
${ }^{200} \mathrm{Po}$ & 955.8 & -26.05 & -25.00 & 843.48 & -34.55 & 1.31 & 2.01 & $1.10^{\mathrm{b}}$ \\
${ }^{202} \mathrm{Po}$ & 942.1 & -29.06 & -28.46 & 236.30 & -20.63 & 1.09 & 2.28 & $1.10^{\mathrm{b}}$ \\
${ }^{204} \mathrm{Po}$ & 810.4 & 0.00 & -21.09 & 100.00 & -5.00 & 0.50 & $2.28^{\mathrm{c}}$ & $1.10^{\mathrm{b}}$ \\
${ }^{206} \mathrm{Po}$ & 717.1 & 0.00 & -4.30 & 100.00 & -5.00 & 0.50 & $2.28^{\mathrm{c}}$ & $1.10^{\mathrm{b}}$ \\
${ }^{208} \mathrm{Po}$ & 643.4 & 0.00 & 6.52 & 100.00 & -5.00 & 0.50 & $2.28^{\mathrm{c}}$ & $1.10^{\mathrm{b}}$ \\
\end{tabular}

aThe effective charges have been taken to be the same as the corresponding values obtained for ${ }^{194} \mathrm{Po}$.

${ }^{\mathrm{b}} e_{N+2}$ corresponding to ${ }^{198} \mathrm{Po}$.

${ }^{c} e_{N}$ corresponding to ${ }^{202} \mathrm{Po}$.

corresponding $\sigma$ values. We stress that the $\sigma$ values do not correspond to experimental error bars, but they are related with the expected accuracy of the IBM-CM calculation to reproduce a particular experimental data point. Thus, they act as a guide so that a given calculated level converges towards the corresponding experimental level. The $\sigma(0.1 \mathrm{keV})$ value for the $2_{1}^{+}$state guarantees the exact reproduction of this experimental most important excitation energy; i.e., the whole energy spectrum is normalized to this experimental energy. The states $4_{1}^{+}, 0_{2}^{+}$, and $2_{2}^{+}$are considered as the most important ones to be reproduced $(\sigma=1 \mathrm{keV})$. The group of states $2_{3}^{+}, 3_{1}^{+}$, $4_{2}^{+}, 6_{1}^{+}$, and $8_{1}^{+}(\sigma=10 \mathrm{keV})$ and $6_{2}^{+}(\sigma=100 \mathrm{keV})$ should also be well reproduced by the calculation to guarantee a correct moment of inertia for the yrast band and the structure of the $0_{2}^{+}$band. Note that we only considered states in the fit with angular momentum and parity unambiguously determined.

In the case of the $E 2$ transitions rates, we have used the available experimental data involving the states presented in Table I, restricted to those $E 2$ transitions for which absolute $B(E 2)$ values are known, except if serious hints that the states involved present noncollective degrees of freedom exist. Additionally, we have taken a value of $\sigma$ that corresponds to $10 \%$ of the $B(E 2)$ values or to the experimental error bar if larger, except for the transition $2_{1}^{+} \rightarrow 0_{1}^{+}$, where a smaller value of $\sigma(0.1$ W.u.) was taken, thereby normalizing, in most of cases, our calculated values to the experimental $B\left(E 2 ; 2_{1}^{+} \rightarrow 0_{1}^{+}\right)$value.

This has resulted in the values of the parameters for the IBM-CM Hamiltonian, as given in Table II. In the cases of ${ }^{190-192} \mathrm{Po}$ and ${ }^{200-208} \mathrm{Po}$, the value of the effective charges, or part of them, cannot be determined because not a single absolute $B(E 2)$ value is known or $\chi^{2}$ is insensitive to their values. However, for completeness we have taken the effective charges of ${ }^{194} \mathrm{Po}$ for ${ }^{190-192} \mathrm{Po}, e_{N+2}$ of ${ }^{198} \mathrm{Po}$ for ${ }^{200-208} \mathrm{Po}$, and $e_{N}$ of ${ }^{202} \mathrm{Po}$ for ${ }^{204-208} \mathrm{Po}$. 


\section{Correlation energy in the configuration mixing approach}

Intruder states are expected to appear, in principle, at an excitation energy well above the corresponding regular ones with identical angular momentum. The reason is that these configurations are related to the creation of a $2 \mathrm{p}-2 \mathrm{~h}$ excitation across the $Z=82$ closed shell. In the case of Po this energy would correspond to $\Delta^{N+2}=2400 \mathrm{keV}$. However, according to Ref. [49], the single-particle energy cost has to be corrected because of the strong pairing energy gain when forming two extra $0^{+}$coupled (particle and hole) pairs, by the quadrupole energy gain when opening up the proton shell, as well as by the monopole correction caused by a change in the single-particle energy gap at $Z=82$ as a function of the neutron number. In particular, around the midshell point at $N=104$, where the number of active nucleons becomes maximal, the energy gain owing to the strong correlation energy is such that the energy of the intruder configurations becomes close to the energy of the regular ones. It may even be that a crossing results, making the intruder state form a ground state. For instance, in the case of Pt isotopes, the nuclei around the midshell point at $N=104$ exhibit a ground state of intruder nature [57,58].

The experimental states can present a strong mixing because of the interaction between both families of configurations (regular and intruder). Therefore, it is not simple in many cases to find out which configurations are dominant in the ground-state wave function. To take advantage of the IBM-CM calculations, we calculate explicitly the "absolute" energy of the lowest $0^{+}$state belonging to both the regular $[N]$ and $[N+2]$ intruder configuration spaces, turning off the interaction among the two families. We choose as the reference energy the energy of the regular Hamiltonian describing the $[N]$ space for the ${ }^{210}$ Po nucleus. This nucleus only consists of a single boson, with the $s$-boson as the lowest energy state, resulting in the zero-energy line (horizontal dashed red line in Fig. 2). The energy of the lowest $0^{+}$state in the regular configuration space $[N], E\left(0_{1}^{+}, N\right)$, is lowered with respect to the reference energy because of the correlation energy and is described by the wave function $\Psi\left(0_{1}^{+}\right)_{N}^{\text {reg }}$ [see also expression (9)]. The lowering depends on the number of bosons. However, the energy of the lowest $0^{+}$state in the intruder configuration space $[N+2], E\left(0_{1}^{+}, N+2\right)$, is described by the wave function $\Psi\left(0_{1}^{+}\right)_{N+2}^{\text {int }}$ [see also expression (10)] and appears at the energy corresponding to $\Delta^{N+2}$. This energy will subsequently be lowered by its specific correlation energy, too. In most cases, the regular configuration with $N$ bosons corresponds to a spherical or slightly deformed shape, while the intruder ones, with $N+2$ bosons, correspond to a more deformed shape. Therefore, the energy gain for the lowest intruder state, described by the wave function $\Psi\left(0_{1}^{+}\right)_{N+2}^{\text {int }}$, use to be larger than for the lowest regular state, described by the wave function $\Psi\left(0_{1}^{+}\right)_{N}^{\mathrm{reg}}$. The relative positions of these lowest regular and intruder states are plotted in Fig. 2. Here it can be clearly appreciated how the energies of both configurations can become very close, depending on the balance between the off-set, $\Delta^{N+2}$, and the difference in the correlation energy $E(N+2)^{\text {corr }}-E(N)^{\text {corr }}$.

One notices how the energies become really close near midshell $(N=106, A=190)$, where the number of active

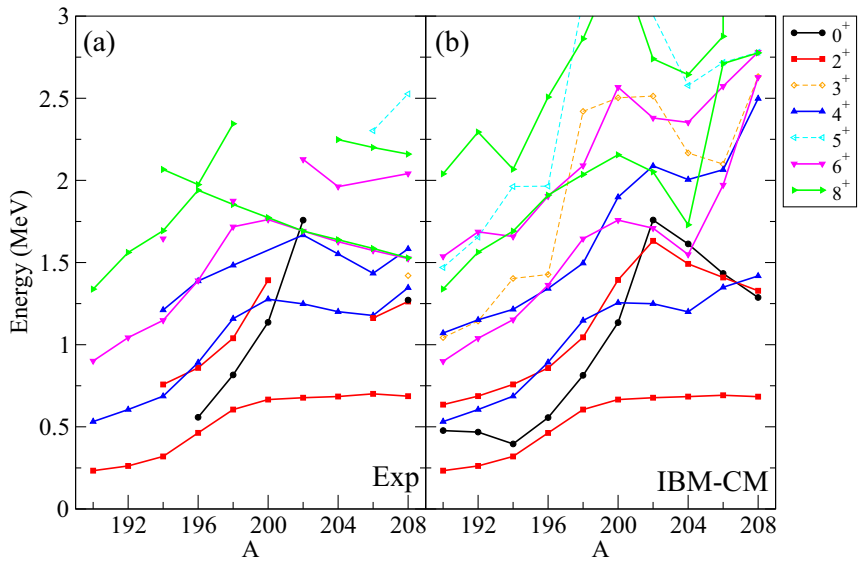

FIG. 3. (Color online) Experimental excitation energies (up to $E_{x} \approx 3.0 \mathrm{MeV}$ ) (a) and the theoretical results (b), obtained from the IBM-CM.

nucleons is maximal, showing that the lowest intruder state can determine the character of the lowest $0_{1}^{+}$state. From $A=196$, and moving towards the heavier masses, the energy difference starts to increase and both states appear to be very well separated. Note that once the isotopes approach the end of the shell, at $N=126$, the number of active bosons is drastically reduced and, therefore, the correlation energy is reduced dramatically. In particular, the energy of the regular state reaches the reference energy, which means that it corresponds to essentially a spherical shape, while the energy of the intruder state approaches the energy $\Delta^{N+2}$ quite closely. Consequently, at the end (or at the beginning) of the shell, the maximum energy difference between both states will correspond to $\Delta^{N+2}$. The existence of a ceiling for the energy difference modulates the parabolic behavior of the energy systematics of the intruder states, transforming it into a flat shape and, therefore, leading to an energy for the intruder states lower than experimentally observed. Clearly, this is a deficiency of the IBM-CM calculations near the shell closure.

\section{Detailed comparison between the experimental data and the IBM-CM results: Energy spectra and electric quadrupole properties}

In this section, we compare in detail the experimental energy spectra with the theoretical ones up to an excitation energy below $E_{x} \approx 3.0 \mathrm{MeV}$. In Fig. 3(a) we plot the experimental data set, while in Fig. 3(b) the theoretical values are depicted. Note that the energy corresponding to the $2_{1}^{+}$ state perfectly agrees with the theoretical one because this level was used to normalize the energies; in other words, we used in the fitting procedure for this level a very small value for $\sigma(0.1 \mathrm{keV})$ to exactly reproduce the $2_{1}^{+}$experimental energy. Comparing the energy spectra, one can distinguish two regions, $A<200$ and $A \geqslant 200$, with an overall better agreement in the first region as compared to the second. A most probable reason for this is the fact that in the lightest isotopes the number of active bosons is substantially larger and the collectivity is enhanced. As we discuss later, in the heavier Po isotopes, noncollective broken-pair states (with a 


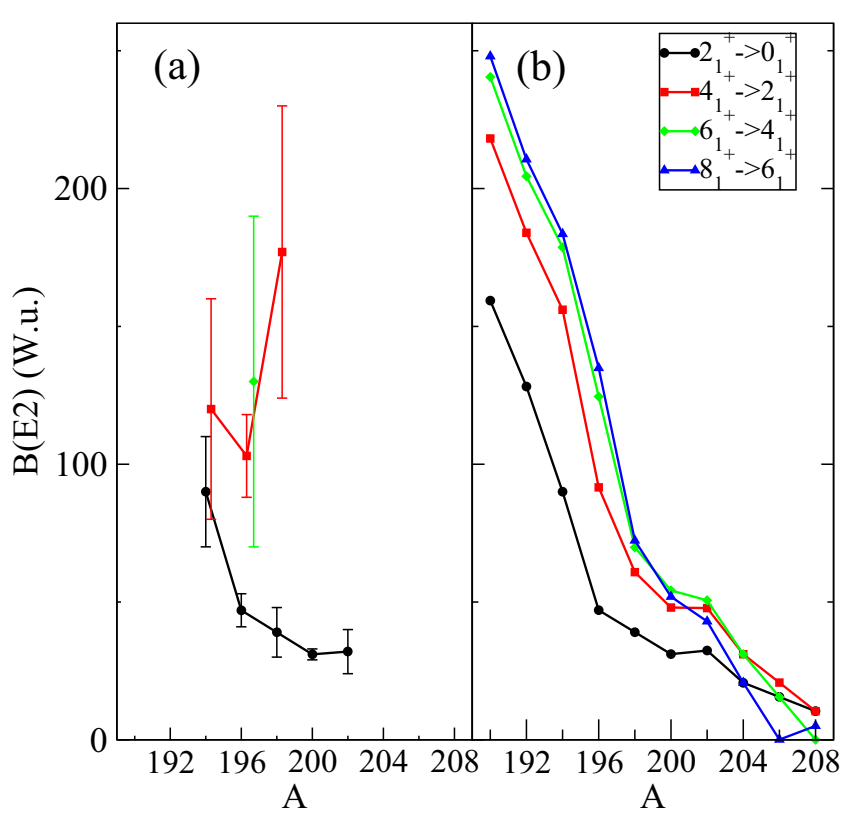

FIG. 4. (Color online) Comparison of the absolute $B(E 2)$ reduced transition probabilities along the yrast band, given in W.u. Panel (a) corresponds to known experimental data and panel (b) to the theoretical IBM-CM results.

$v=2$ seniority character) do appear in the low-energy part of the energy spectrum. Those states are not well reproduced within the IBM space because of the restriction to a set of interacting $s$ and $d$ bosons.

One observes in Fig. 3 how the compression of the energy spectrum, with decreasing mass number, is well reproduced. The same happens for the systematic dropping of the energy of the $0_{2}^{+}$states, and the energy of the yrast band, more generally. Note that the agreement is better for those states with low and even angular momenta.

Next, we carry out a comparison for the $B(E 2)$ values, which is a much more stringent test than the excitation energy, because these numbers are highly dependent on the detailed structure of the wave function. Although the existing experimental information on $B(E 2)$ values is rather scarce, recent new results from Coulomb excitation experiments at REX-ISOLDE, in particular for the ${ }^{196-198}$ Po isotopes, have appeared [115], improving the experimental knowledge of this mass region.

In Figs. 4 and 5 we compare the $B(E 2)$ reduced transition probabilities, while in Fig. 6 we compare the electric quadrupole moments. We also present a more detailed comparison on $B(E 2)$ values in Table III.

In Fig. 4 we present the intraband $B(E 2)$ values along the yrast band, for which the most complete experimental information exists. In a similar way as for the excitation energies, we used the $B\left(E 2 ; 2_{1}^{+} \rightarrow 0_{1}^{+}\right)$reduced transition probability to normalize the theoretical results. Note that for some nuclei, where experimental information is scarce, we have used the corresponding values of the neighboring isotopes to determine the effective charges (as explained in Sec. IV B). On the experimental side, the value of $B\left(E 2 ; 2_{1}^{+} \rightarrow 0_{1}^{+}\right)$is

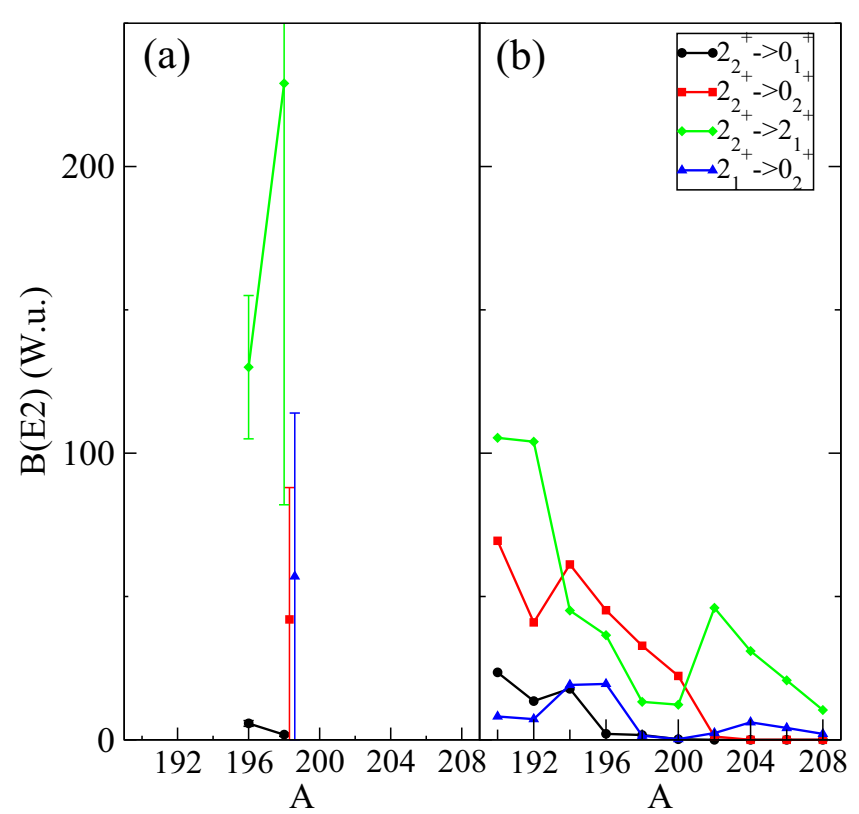

FIG. 5. (Color online) Comparison of the few nonyrast intraband absolute $B(E 2)$ reduced transition probabilities, given in W.u. Panel (a) corresponds to the few known experimental data, panel (b) to the theoretical IBM-CM results.

systematically dropping, which denotes a reduction of the collectivity of the states when approaching the end of the neutron shell. However, this is not the case for $B\left(E 2 ; 4_{1}^{+} \rightarrow\right.$ $2_{1}^{+}$) in ${ }^{198} \mathrm{Po}$, where an unexpected large value is observed.

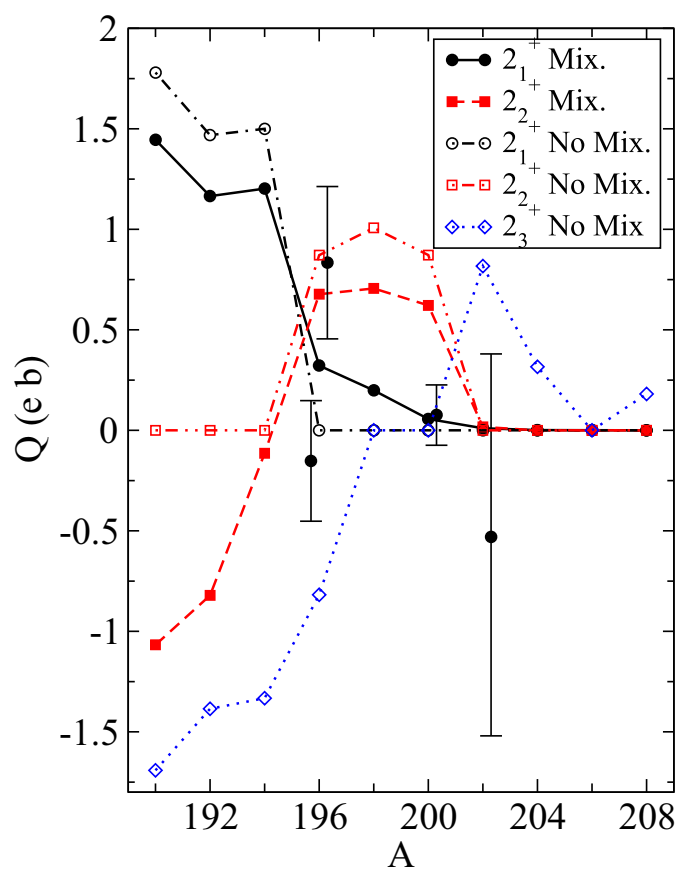

FIG. 6. (Color online) IBM-CM values of the quadrupole moments for the $2_{1}^{+}$and $2_{2}^{+}$states in ${ }^{190-208} \mathrm{Po}$. The quadrupole moments are given in units $e \mathrm{~b}$. The dash-dotted and dotted lines indicate the quadrupole moments when the mixing Hamiltonian is switched off, corresponding to the unperturbed $2_{1}^{+}, 2_{2}^{+}$, and $2_{3}^{+}$states. 
TABLE III. Comparison of the experimental absolute $B(E 2)$ values (given in units of W.u.) with the IBM-CM Hamiltonian results. Data are taken from the Nuclear Data Sheets [73-78], complemented with references presented in Sec. II.

\begin{tabular}{lccc}
\hline \hline Isotope & Transition & Experiment & IBM-CM \\
\hline${ }^{194} \mathrm{Po}$ & $2_{1}^{+} \rightarrow 0_{1}^{+}$ & $90(20)$ & 90 \\
& $4_{1}^{+} \rightarrow 2_{1}^{+}$ & $120(40)$ & 156 \\
${ }^{196} \mathrm{Po}$ & $2_{1}^{+} \rightarrow 0_{1}^{+}$ & $47(6)$ & 47 \\
& $4_{1}^{+} \rightarrow 2_{1}^{+}$ & $103(15)$ & 92 \\
& $6_{1}^{+} \rightarrow 4_{1}^{+}$ & $130(60)$ & 125 \\
& $2_{2}^{+} \rightarrow 0_{1}^{+}$ & $5.7(10)^{\mathrm{a}}$ & 2.1 \\
& $2_{2}^{+} \rightarrow 2_{1}^{+}$ & $130(25)^{\mathrm{a}}$ & 36 \\
${ }^{198} \mathrm{Po}$ & $2_{1}^{+} \rightarrow 0_{1}^{+}$ & $39(9)$ & 39 \\
& $4_{1}^{+} \rightarrow 2_{1}^{+}$ & $177(53)$ & 61 \\
& $8_{1}^{+} \rightarrow 6_{1}^{+}$ & $2.0(1)^{\mathrm{b}, \mathrm{c}}$ & 72 \\
& $0_{2}^{+} \rightarrow 2_{1}^{+}$ & $285\left(_{-285}^{+980}\right)^{\mathrm{a}}$ & 7 \\
& $2_{2}^{+} \rightarrow 0_{1}^{+}$ & $1.8\left(_{-0.6}^{+1.6}\right)^{\mathrm{a}}$ & 1.7 \\
& $2_{2}^{+} \rightarrow 2_{1}^{+}$ & $229(147)^{\mathrm{a}}$ & 13 \\
& $2_{2}^{+} \rightarrow 0_{2}^{+}$ & $42(56)^{\mathrm{a}}$ & 33 \\
${ }^{200} \mathrm{Po} \mathrm{Po}$ & $2_{1}^{+} \rightarrow 0_{1}^{+}$ & $31(2)$ & 31 \\
& $8_{1}^{+} \rightarrow 6_{1}^{+}$ & $9.4(5)^{\mathrm{b}}$ & 52 \\
\hline \hline
\end{tabular}

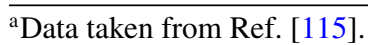

${ }^{\mathrm{b}}$ Experimental data not included in the fit.

${ }^{\mathrm{c}}$ Data taken from Ref. [91].

However, the theoretical results show a continuous drop of all the intraband transitions, resulting from the reduction of the collectivity of the states (in line with a decreasing number of active bosons).
In Fig. 5 we study the systematics of some interband transitions involving the states $0_{1,2}^{+}$and $2_{1,2}^{+}$. Because of the lack of experimental data and the large error bars, it is difficult to extract any trend. Regarding the theoretical results, once more, one notices an overall reduction of the $B(E 2)$ values. We point out that for $A>196$, the observed $B(E 2)$ values are of the same order as the observed $B(E 2)$ values in the yrast band. This represents a hint about the changing mixing character of the states as a function of mass number $A$ (see Fig. 12 as an illustration of the changing structure of the wave function).

A different way to extract information on the changing character of the wave functions for the lowest two $2^{+}$states is comparing the spectroscopic quadrupole moments, shown in Fig. 6. Here, we compare the theoretical and the experimental values of the quadrupole moment for the states $2_{1}^{+}$and $2_{2}^{+}$. Moreover, we present the values corresponding to the unperturbed lowest-lying three $2^{+}$states. One notices that the $2_{1}^{+}$state corresponds to a well deformed and oblate shape for the lightest isotopes, which is smoothly changing into a rather spherical shape for $A=202$ and onwards. However, the $2_{2}^{+}$ state corresponds to a prolate shape for the lightest isotopes, changing into an oblate shape for $A=196-200$ and into a spherical shape for $A=202$ and the still heavier isotopes. When comparing with the unperturbed values, one can see how the $2_{1}^{+}$state is built up as a mixture of the first two unperturbed $2^{+}$states. However, to understand the second $2_{2}^{+}$state, we have to resort to a mixture of the second and third unperturbed $2^{+}$states mainly. This figure presents a clear proof of the changing character for the first two $2^{+}$states as a function of mass number. This issue is discussed in Sec. IV $\mathrm{E}$ in a more quantitative way.

In Figs. 7 and 8 we present the experimental and theoretical energy spectra (up to $E_{x} \approx 2.5 \mathrm{MeV}$ ) for masses $A=190-198$, which is the region where the coexistence should be more evident. We include in the comparison the known absolute $B(E 2)$ values. One observes a rather distinct

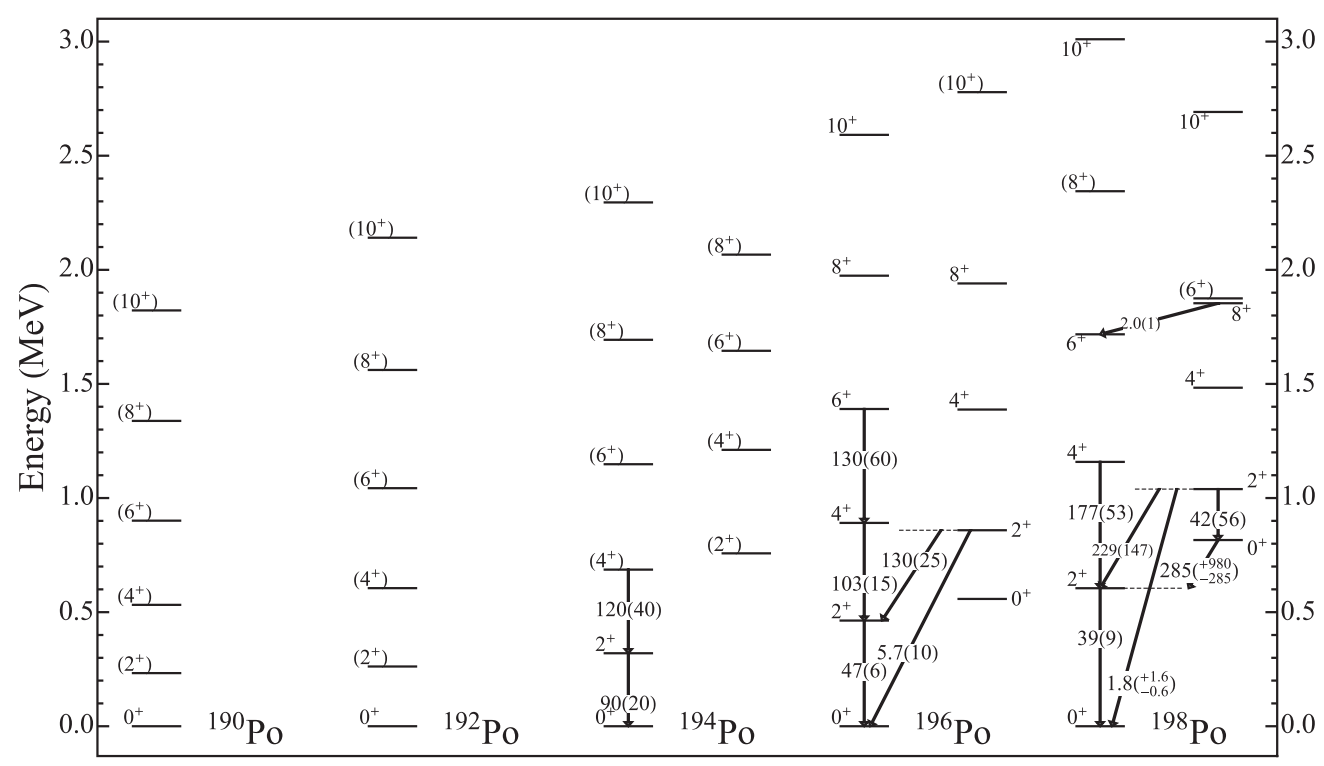

FIG. 7. Experimental excitation energies and absolute $B(E 2)$ transition rates for selected states in ${ }^{190-198}$ Po. 


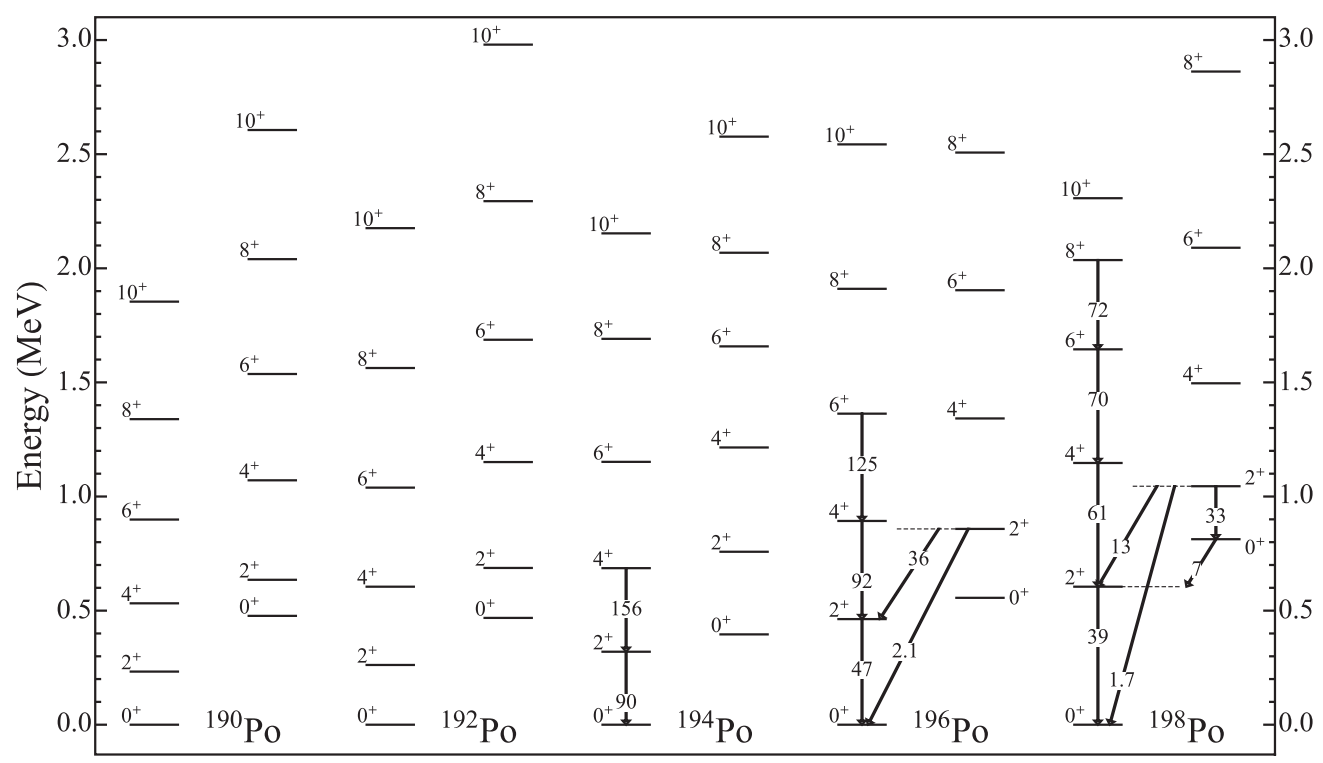

FIG. 8. Theoretical excitation energies and absolute $B(E 2)$ transition rates for selected states in ${ }^{190-198}$ Po.

rotational structure (along the yrast band) for $A=190-194$, followed by a structure that is changing into a more vibrational behavior for masses $A=196-198$.

The Po energy spectra show some other most interesting structural changes starting at the neutron $N=126$ shell closure, moving towards the neutron-deficient region (decreasing towards midshell at $A=188$ ) (see Figs. 1 and 3). One starts with the typical $\pi\left(1 h_{9 / 2}\right)^{2}$ two-particle spectrum governed by the seniority characteristics of the two-body proton-proton interaction. An energy gap of $\sim 0.8 \mathrm{MeV}$ is observed from the $8_{1}^{+}$to the $8_{2}^{+}$state energy difference (see Fig. 1), which most probably results from a $\pi\left(1 h_{9 / 2} 2 f_{7 / 2}\right)$ configuration, as a measure of the single-particle energy gap. Next, one observes the remaining characteristics of a seniority $v=2$ spectrum, albeit with an immediate drop of the $2_{1}^{+}$state, when removing two neutrons from the closed $N=126$ shell, which then keeps a remarkably constant energy down to mass $A=200$ $(N=116)$. The energy of the $4_{1}^{+}$slightly drops but also remains at an approximate constant energy. The higher spin members $6_{1}^{+}, 8_{1}^{+}$are shifted upwards in a rather regular way down to mass $A=198(N=114)$, before first the $\sigma_{1}^{+}$starts dropping systematically down in excitation energy at $A=196$ $(N=112)$, followed by the $8_{1}^{+}$dropping too from $A=194$ $(N=110)$ (see Figs. 1 and 3$)$. Therefore, it looks like the region $208 \geqslant A \geqslant 198$ is reminiscent of a vibrational pattern where the specific two-nucleon properties of the high-spin proton pair remain rather well intact down to the lower value at $A=198$. An interesting test to gain deeper insight into this region, and the connection to the yet lower-mass region $196 \geqslant A \geqslant 190$ where a dominant rotational-like and more collective pattern is showing up, can be derived from a study of, e.g., the $B\left(E 2 ; 8_{1}^{+} \rightarrow 6_{1}^{+}\right)$value, as well as, of the $g$-factor and the quadrupole moment of the high-spin $8_{1}^{+}$state (see Table III and Fig. 9). Experiments [145,146] have shown that the $g$ factor stays remarkably constant, going down to $A=198$, at a value consistent with the $\pi\left(1 h_{9 / 2}\right)^{2} 8^{+}$ configuration (for a pure $v=2$ configuration, as a function of $n$, the number of holes in the $\pi\left(1 h_{9 / 2}\right)$ orbital, the $g$ factor remains constant), indicating a minor influence of collective admixtures into this state. Maj et al. [91] have analyzed the $B\left(E 2 ; 8_{1}^{+} \rightarrow 6_{1}^{+}\right)$value as well as the $8_{1}^{+}$quadrupole moment to extract an effective charge (see, e.g., Fig. 9 in Ref. [91]). The influence of particle-core coupling on the quadrupole moment of the $8_{1}^{+}$state has been studied for the Po nuclei with $A=202$ and 204, indicating an increase in the quadrupole moment as low as $A=200$ [147]. The effective charge shows a steady increase of $\sim 2$ from $A=210$ down to mass $A=200$, from which a sharp drop is observed moving towards $A=198$. This seems to support the idea that, in particular for the high-spin members with a seniority $v=2$ character, those states only couple weakly with collective quadrupole excitations. Moreover, these results also corroborate the fact that from $A=196$ and onwards with decreasing $A$ value,
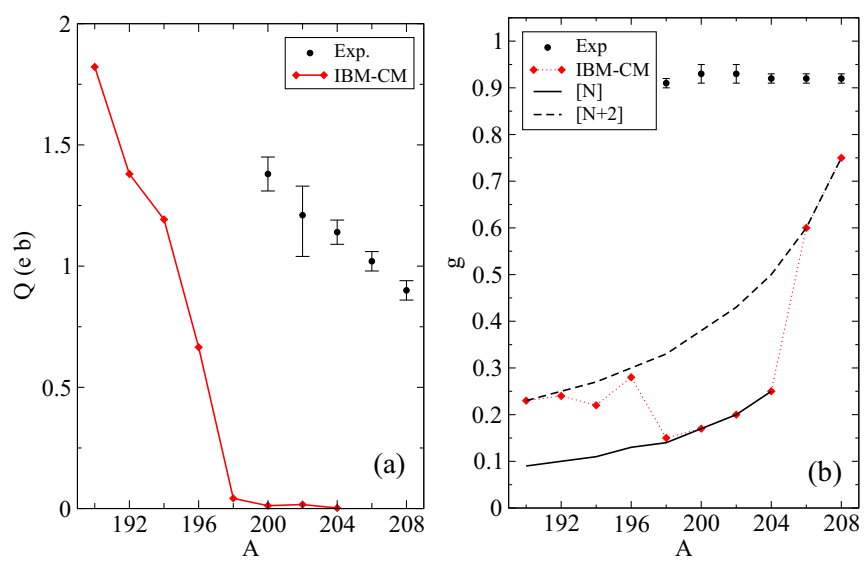

FIG. 9. (Color online) (a) Experimental and theoretical quadrupole moment for the $8_{1}^{+}$state (note that the theoretical values for $A=206$ and 208 are not shown owing to the reduced number of valence bosons). (b) Gyromagnetic factor for the $8_{1}^{+}$state (experimental data and theoretical results). 
large collective components should appear in the $6_{1}^{+}$state, even though the $g$ factor of the $8_{1}^{+}$remains constant between $A=200$ and $A=198[145,146]$. In view of the discrepancies between the IBM-CM calculations and the experimental data concerning the $g$ factor and the $B\left(E 2 ; 8_{1}^{+} \rightarrow 6_{1}^{+}\right)$value, those states should have a very weak coupling with the quadrupole collective excitations and genuinely correspond to almost pure $v=2$ seniority configurations (see Fig. 9).

Finally, it is worth noting a recent beyond-mean-field (BMF) calculation performed for the lead region by Yao et al. [127] and, in particular, with results for the even-even Po isotopes $(A=186-204)$. They have calculated energy spectra (general systematics), as well as charge radii, $\rho^{2}\left(0_{2}^{+} \rightarrow 0_{1}^{+}\right)$, quadrupole moments for the lowest $2^{+}$and $4^{+}$states, and $B\left(E 2 ; 2_{k}^{+} \rightarrow 0_{l}^{+}\right)$(with $\left.k, l=1,2\right)$. Comparisons with existing data have also been carried out for ${ }^{190} \mathrm{Po}$ and ${ }^{194} \mathrm{Po}$ in Ref. [113] and for ${ }^{196}$ Po in Ref. [114]). Because their formulation is within a mean-field context, invoking the concept of nuclear shapes (spherical, oblate, prolate), it is not always possible to compare with the present IBM-CM approach in which the model space is restricted to $0 \mathrm{p}-0 \mathrm{~h}$ and $2 \mathrm{p}-2 \mathrm{~h}$ excitations across the $Z=82$ closed shell. A few general remarks are in place. Regarding the energy spectra the main conclusion of the calculations are the overestimation of the $0_{2}^{+}$energy (which is predicted to be oblate), though the slope of the variation with respect to $A$ is well reproduced and, moreover, that the calculation is not able to reproduce the almost constant value of the $2_{1}^{+}$excitation energy, giving rise to a steady increase of the energy as a function of $A$. Another very interesting outcome of the calculation is the spectroscopic quadrupole moment (note that in Ref. [127] an equivalent magnitude is provided, $\beta_{s}$ ) for the $2_{1}^{+}$and $2_{2}^{+}$states. The calculation predicts a prolate shape for the $2_{1}^{+}$state while oblate for the $2_{2}^{+}$state up to $N=106(A=190)$, interchanging at this point their character, becoming almost spherical (but slightly oblate) for $N=114(A=198)$ and onwards. In the case of $A=190$, the calculation exhibits the presence of a prolate and an oblate band structure, very much like the IBM-CM model results. The results for the charge radii give an overall correct trend. However, the upsloping trend in $\left\langle r^{2}\right\rangle$ (relative to the value for $\left.{ }^{210} \mathrm{Po}\right)$, starting at $N=112(A=196)$, is not well described within the BMF description as compared with the IBM-CM.

\section{E. Wave-function structure: From the unperturbed structure to configuration mixing}

We start our analysis with the structure of the configurationmixed wave functions along the yrast levels, expressed as a function of the $[N]$ and $[N+2]$ basis states, as given in Eq. (7). In Figs. 10(a) and 10(b), we present the weight of the wave functions contained within the $[N]$-boson subspace, defined as the sum of the squared amplitudes $w^{k}(J, N) \equiv$ $\sum_{i}\left|a_{i}^{k}(J ; N)\right|^{2}$, for both the yrast states, $k=1$, and the $k=2$ states (the latter are indicated with a dashed line) for spins $J^{\pi}=0^{+}, 2^{+}, 3^{+}, 4^{+}$in panel (a) and $J=5^{+}, 6^{+}, 7^{+}, 8^{+}$in panel (b). The results exhibit an interesting behavior: The yrast states show rather an intruder character for mass $A=190$ that quickly is changing into a regular one in the mass interval $A=194-200$. For the $0^{+}$states, they are fully mixed for

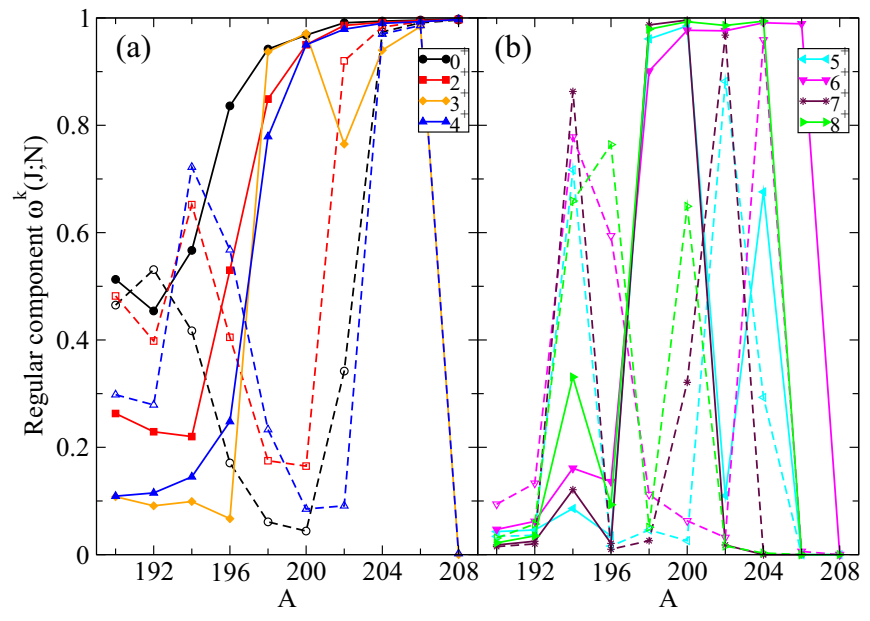

FIG. 10. (Color online) Regular content of the two lowest-lying states for each $J$ value (solid lines with solid symbols correspond with the first state, while dashed lines with open symbols correspond with the second state) resulting from the IBM-CM calculation, as presented in Fig. 3.

$A=190$ and, subsequently, the $0_{1}^{+}$state is changing into a pure regular one, while the character of the $0_{2}^{+}$state is essentially purely intruder for $A=200$, later on changing into a pure regular character for $A=204$. The behavior for the $2^{+}$and $4^{+}$states is very similar. The first $\left(2^{+}\right.$or $\left.4^{+}\right)$state starts as a dominant intruder state for the lighter isotopes, steadily changing with an increase of its regular component, becoming a fully regular configuration for $A=200$ and onwards to the heavier masses. The second $2^{+}$and $4^{+}$states start with a regular component of $\approx 50 \%$ and $\approx 30 \%$, respectively, reaching a maximal value for $A=194(\approx 70 \%)$, then decreasing when moving to $A=200$, increasing up to reach a purely regular character by mass $A=202$. Indeed, the $0_{2}^{+}, 2_{2}^{+}$, and $4_{2}^{+}$ states exhibit a similar trend. The other states exhibit a more erratic behavior, mainly because of the many crossings in the unperturbed energy spectra of the regular and intruder states, although appearing at very similar energies. Finally, note that the results for $A=208$ should all correspond to pure regular states because the intruder states appear at a much higher energy at $N=126$. Because of the constraint put on the value of $\Delta^{N+2}$ (see the discussion in Sec. IV C), the intruder states appear too low in the energy spectra. Moreover, because of the reduced number of bosons in ${ }^{208} \mathrm{Po}(Z=84, N=124)$, two, only regular states up to spin $J=4$ can be constructed within the IBM. Consequently, it is not possible to describe the higher-spin ( $J=8$ and beyond) states showing up in the mass region $204 \leqslant A \leqslant 208$ in a reasonable way within the IBM, in view of the specific high-spin broken-pair states appearing at $\approx 1.5-2 \mathrm{MeV}$.

Regarding the energy systematics of the intruder states, one expects a parabolic shape centered around $N=104$, as is the case of $\mathrm{Hg}$ and $\mathrm{Pb}$. However, this is not the case for Po or for Pt isotopes. The reason for not observing the parabolic shape is the rather strong interaction between regular and intruder configurations and the crossing of the intruder and regular states in the ground state. Therefore, it is very 


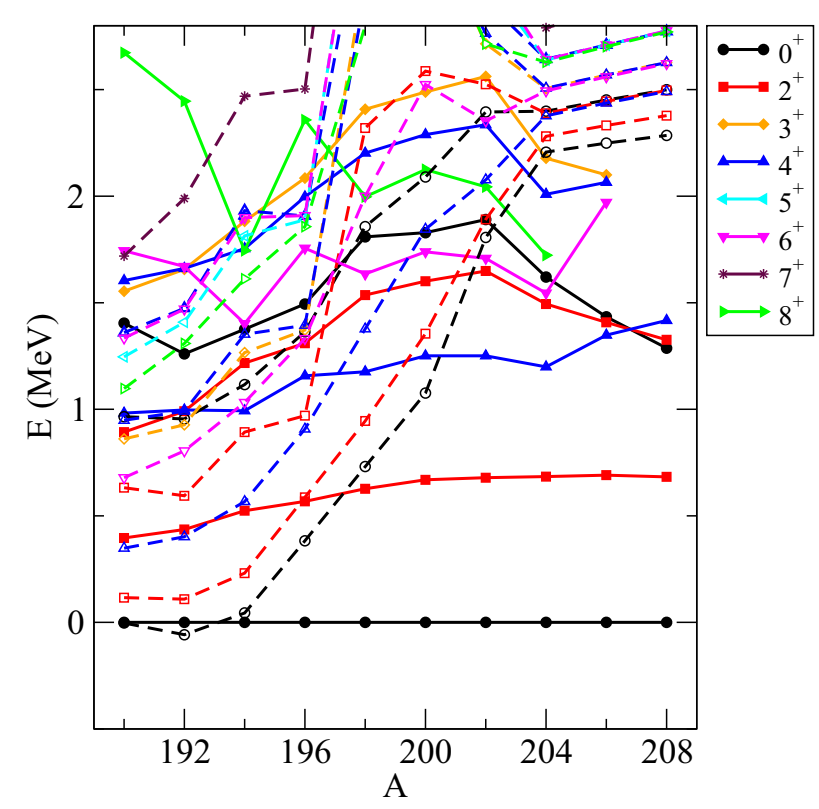

FIG. 11. (Color online) Energy spectra for the IBM-CM Hamiltonian presented in Table II, switching off the mixing term. The two lowest-lying regular states and the lowest-lying intruder state for each of the angular momenta are shown (solid lines with solid symbols for the regular states while dashed lines with open symbols are used for the intruder ones).

enlightening to calculate the energy spectra as a function of $A$ switching off the mixing part of the Hamiltonian, i.e., keeping $w_{0}^{N, N+2}=w_{2}^{N, N+2}=0$. These spectra are depicted in Fig. 11, where we show the lowest two regular and the lowest two intruder states for different angular momenta. One observes a rather flat behavior of the energy for the regular states, still indicating a downsloping tendency when moving towards the lighter Po isotopes. The energy of the intruder states is smoothly decreasing down to neutron midshell $(N=106)$. In this region, the parabola becomes very flat $(A=190-194)$. This results mainly from the smooth change of the Hamiltonian parameters when passing from isotope to isotope. A striking fact is the almost degeneracy of the unperturbed regular and intruder $0^{+}$states for $A=190-194$, with the intruder configuration becoming the lowest one in the energy spectrum for $A=190-192$. The crossing of a regular and a intruder configuration with the same angular momentum has a strong influence on the regular component of the states resulting from the full IBM-CM calculation (see Fig. 10) inducing an interchange of character between the two states. In the situation of the $J^{\pi}=2^{+}$unperturbed energy spectrum, the closest approach happens at $A=196$ and in Fig. 10 one notices that the $2_{1,2}^{+}$states interchange their character at this point. The same happens for the $4^{+}$states at $A=196-198$.

A most interesting decomposition of the wave function is obtained by first calculating the wave functions within the $N$ subspace as

$$
\Psi(l, J M)_{N}^{\mathrm{reg}}=\sum_{i} c_{i}^{l}(J ; N) \psi\left((s d)_{i}^{N} ; J M\right)
$$
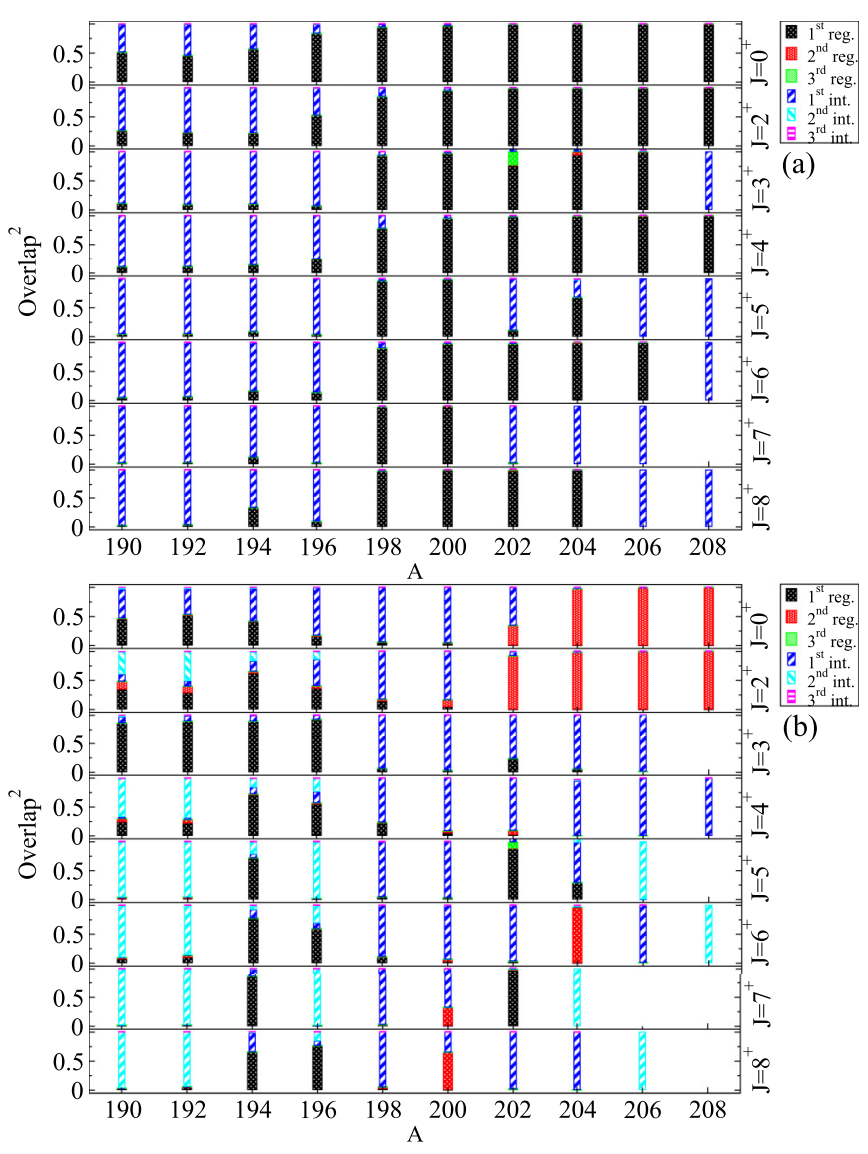

FIG. 12. (Color online) Overlap of the wave functions of Eq. (7), with the wave functions describing the unperturbed basis Eqs. (9) and (10). (a) Overlaps for first $0^{+}, 2^{+}, 3^{+}, 4^{+} ; 5^{+}, 6^{+}, 7^{+}, 8^{+}$state; (b) overlaps for the corresponding second state (see also text).

and likewise for the intruder (or $N+2$ subspace) as

$$
\Psi(m, J M)_{N+2}^{\mathrm{int}}=\sum_{j} c_{j}^{m}(J ; N+2) \psi\left((s d)_{j}^{N+2} ; J M\right),
$$

defining an "intermediate" basis $[53,54]$. This generates a set of bands within the $0 \mathrm{p}-0 \mathrm{~h}$ and $2 \mathrm{p}-2 \mathrm{~h}$ subspaces, corresponding to the unperturbed bands that are extracted in schematic twolevel phenomenological model calculations (as discussed in references [100,137-139,148-152]), and indeed correspond to the unperturbed energy levels depicted in Fig. 11.

The overlaps ${ }_{N}\langle l, J M \mid k, J M\rangle$ and ${ }_{N+2}\langle m, J M \mid k, J M\rangle$ can then be expressed as

$$
{ }_{N}\langle l, J M \mid k, J M\rangle=\sum_{i} a_{i}^{k}(J ; N) c_{i}^{l}(J ; N)
$$

and

${ }_{N+2}\langle m, J M \mid k, J M\rangle=\sum_{j} b_{j}^{k}(J ; N+2) c_{j}^{m}(J ; N+2)$

[see expressions (9) and (10)]. In Fig. 12 we show these overlaps, but squared, where we restrict ourselves to the first and second state $(k=1,2)$ with angular momentum $J^{\pi}=0^{+}$, $2^{+}, 3^{+}, 4^{+}, 5^{+}, 6^{+}, 7^{+}, 8^{+}$, and give the overlaps with the lowest three bands within the regular $(N)$ and intruder $(N+2)$ 
spaces $(l=1,2,3$ and $m=1,2,3)$. Because these figures are given as a function of mass number, one obtains a graphical insight into the changing wave-function content. In panel (a), which corresponds to the first state, one observes for the $0^{+}$state a strong mixing between the first regular and first intruder unmixed states (also called the "intermediate basis") for $A=190-194$, while for the heaviest isotopes the state is mainly of regular character. For the $2^{+}$states the situation is very much the same, though the mixing is much more reduced. For the higher spin states one observes a sharp border at $A=198$, in such a way that for $A \leqslant 196$ the states correspond to the first intruder configuration, while for $A \geqslant 198$ they correspond to the first regular configuration. Note that the intruder character observed for the heavier isotopes is somehow artificial because of the reduced number of bosons and also to the reduced excitation energy of the intruder configurations. In panel (b) the situation becomes rather fuzzy especially for $J>2$. For the $0^{+}$state one observes a mixture between the first regular and intruder configurations up to $A=196$; then the composition changes into a pure first intruder configuration and finally ends up as the second regular configuration. Concerning the $2^{+}$state, the lighter isotopes correspond to a mixture of the second intruder and the first and second regular configuration, the composition changing into the first intruder for $A=198-200$, finally ending as the second regular configuration. For the higher-spin states, the pattern is not so clear. In general, the states become rather pure and the changes in their composition are mainly attributable to the many crossings between the unperturbed regular and intruder configurations.

\section{STUDY OF OTHER OBSERVABLES: $\alpha$-DECAY-HINDRANCE FACTORS AND ISOTOPIC SHIFTS}

\section{A. $\alpha$-decay-hindrance factors}

The $\alpha$-decay process can be used as an important tool to probe nuclear structure; in particular, the overlap factor between the initial and final wave functions has the character of an $\alpha$-particle spectroscopic factor. Because calculations of the absolute decay rate are very difficult, most often one studies decay branches, characterized by a hindrance factor described by the expression

$$
\mathrm{HF}=\frac{\delta_{\mathrm{gs}}^{2}}{\delta_{\mathrm{ex}}^{2}}=\frac{I_{\mathrm{gs}} P_{\mathrm{ex}}}{I_{\mathrm{ex}} P_{\mathrm{gs}}},
$$

where $\delta_{i}^{2}$ is the reduced $\alpha$ width, $P_{\alpha_{i}}$ the penetration probability through the combined Coulomb and centrifugal barrier [106] and $I_{i}$ the $\alpha$-decay intensity (with $i=$ gs, ex for the ground state and excited state, respectively) [106].

The $\mathrm{Pb}$ region has been studied intensively and hindrance factors have been used to obtain valuable information on shape coexistence and mixing of various nuclear shapes. In particular, a two-level mixing model, suggested by Wauters et al. [97], has been used to analyze the experimental hindrance factors and extract complementary information on mixing between different nuclear configurations.

The structure information on the composition of the $0_{1}^{+}$ ground state in the ${ }^{190-198}$ Po nuclei is derived from the

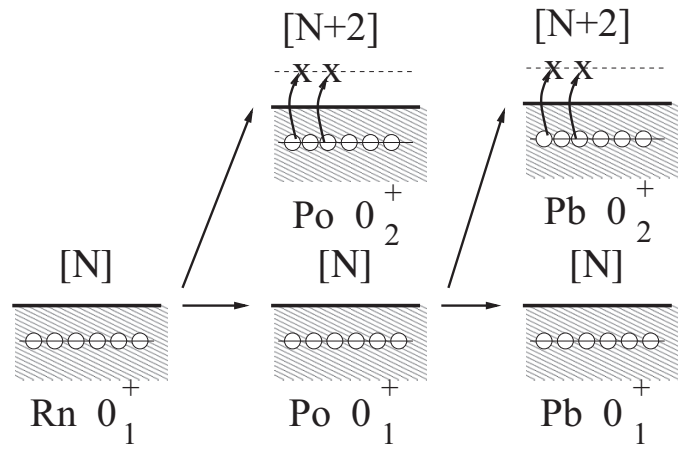

FIG. 13. Schematic view of $\alpha$ decay from the Rn nuclei into the Po nuclei and from the Po nuclei into the $\mathrm{Pb}$ nuclei.

specific $\alpha$-decay-hindrance factors into both the $0_{1}^{+}$and the $0_{2}^{+}$states into the corresponding daughter ${ }^{186-194} \mathrm{~Pb}$ nuclei (see Fig. 13). Use is made of the fact that for the $\mathrm{Pb}$ nuclei, it was shown, through study of the $\mathrm{Pb}$ nuclear charge radii [6] and very weak $E 0$ decay strength from the $0_{2}^{+} \rightarrow 0_{1}^{+}$transition, that the $\mathrm{Pb}$ nuclei retain a spherical ground-state structure. The experimental results on these Po nuclei [95-97,99$101,106,107]$ have been analyzed in these papers, resulting in hindrance factors of $2.8 \pm 0.5,2.5 \pm 0.1,1.1 \pm 0.1$, and 0.4 for $A=198,196,194$, and 192, respectively. The major result is the decreasing trend in these hindrance factors, even favoring decay to the excited $\mathrm{O}_{2}^{+}$excited state at $591 \mathrm{keV}$ in ${ }^{188} \mathrm{~Pb}$ starting from ${ }^{192}$ Po. In those studies, and using a two-state mixing model [97], an intruder character for the ground state $0_{1}^{+}$state in ${ }^{192}$ Po turns out to be $\approx 63 \%$ [100], or, even larger, $>65 \%$ [101]. More recently, $\alpha$ decay from ${ }^{188,192}$ Po has been observed into the final $0^{+}$excited states in ${ }^{184,188} \mathrm{~Pb}$ [104]. Their analyses results in hindrance factors into the first excited $0_{2}^{+}$state of $0.08 \pm 0.03$ and $0.57 \pm 0.12$, respectively, which may well turn out to be an indication of a very large overlap of the wave functions in the initial and excited states in the final nucleus. In that latter paper, an analysis was carried out from inspecting the various energy minima in both the $\mathrm{Po}$ and the $\mathrm{Pb}$ nuclei, obtaining qualitative information on overlaps of the corresponding wave functions, derived from the Nilsson-Strutinsky approach [153]. An analysis of hindrance factors was also carried out using a similar method, making use of deformed mean-field wave functions at the energy minima of the total energy surfaces, pointing out that this approach, albeit different from an approach explicitly including mp-nh excitations across the closed $Z=82$ shell closure, renders quite similar results [154].

The wave functions, obtained from the IBM-CM (see also similar results on the study of the $\mathrm{Pt} \mathrm{[58]} \mathrm{and} \mathrm{Hg}$ [64] nuclei on $\alpha$-decay-hindrance factors), give rise to the following theoretical values of $6 \%, 17 \%, 42 \%$, and $53 \%$, corresponding with the weight factors of the intruder component (see Fig. 10) for the masses $A=198,196,194$, and 192, respectively. These numbers can be compared with the experimental relative hindrance factors as discussed before, with a rather good agreement.

On the other side, $\alpha$-decay starting from the even-even ${ }^{A} \mathrm{Rn}$ nuclei (see Fig. 13), into the ${ }^{A-4}$ Po nuclei, gives very strong 
fingerprints for observing excited $0^{+}$states, in particular, experiments studying the decay of ${ }^{202} \mathrm{Rn}$ into ${ }^{198} \mathrm{Po}$ [94] and of ${ }^{198,200} \mathrm{Rn}$ [92] into the ${ }^{194,196}$ Po nuclei. The analysis of these data, using a two-state mixing model [148], resulted in detailed information on the more deformed intruder configuration in the $2_{1}^{+}$state. Its weight factor is changing from $7 \%$, passing by $31 \%$ and $72 \%$, towards the value of $88 \%$, for $A=200,198,196$, and 194, respectively. It is interesting to compare these results with the present IBM-CM calculations, resulting in a variation of the intruder weight factor of $5 \%, 15 \%, 47 \%$, and $78 \%$ (see Fig. 10) for the same mass values. This again indicates a rather good description of the changing wave function structure for the $2_{1}^{+}$state in the Po even-even nuclei.

Studies on $\alpha$ decay from the odd-mass Po nuclei have been carried out for the same mass region, with now an extra neutron coupled to the underlying Po even-even core $[98,102,103,105]$. These experiments show the presence of rather weakly particle-core coupled states, associated with a spherical configuration for the even-even underlying core, as well the appearance of a more strongly coupled structure, associated with coupling of the odd particle with the more deformed configuration of the even-even core.

A lot of effort has been invested into going beyond the more simplified two-state mixing model that has been extensively studied analyzing the $\alpha$-decay-hindrance factors related to the decay into the excited $0_{i}^{+}$states in the daughter nuclei. In particular, more detailed calculations have been carried out by Delion et al. [155,156], and more recently in Ref. [108], emphasizing the need for a microscopic quasirandom-phase approximation description that encompasses both neutron and proton pairing vibrations as well as including proton $2 \mathrm{p}-2 \mathrm{~h}$ excitations across the $Z=82$ closed shell. More in particular, calculations have mainly concentrated to better understand the observed hindrance factors for $\alpha$ decay, leading into the neutron-deficient $\mathrm{Po}, \mathrm{Pb}, \mathrm{Hg}$, and $\mathrm{Pt}$ nuclei. Karlgren et al. [154] use the information on the energy minima in the $\beta-\gamma$ plane of the total energy surface as the essential input to calculate the hindrance factors. They also discuss a possible connection between the present deformed mean-field approach with a picture in which specific particle-hole excitations are invoked within a shell-model approach. Moreover, $\mathrm{Xu}$ et al. [157] formulates the $\alpha$-decay-hindrance factors within a density-dependent cluster model to describe the tunneling of a preformed cluster through the deformed Coulomb barrier.

\section{B. Isotopic shifts}

Experimental information about ground-state charge radii is also available for both the even-even [118] and the oddmass [116,117] Po nuclei. Combined with similar data for the adjacent $\mathrm{Po}, \mathrm{Pb}$, and $\mathrm{Pt}$ nuclei, as well as for the odd-mass $\mathrm{Bi}, \mathrm{Tl}$, and $\mathrm{Au}$ nuclei, the systematic variation of the charge radii supplies invaluable information on the ground-state wave function [158-160]. We illustrate the overall behavior of $\left\langle r^{2}\right\rangle_{A}$ relative to the radius at mass $A=210$ in Fig. 14(a) and the relative changes defined as $\Delta\left\langle r^{2}\right\rangle_{A} \equiv\left\langle r^{2}\right\rangle_{A+2}-\left\langle r^{2}\right\rangle_{A}$ in Fig. 14(b). The experimental data are taken from Cocolios et al. [118].
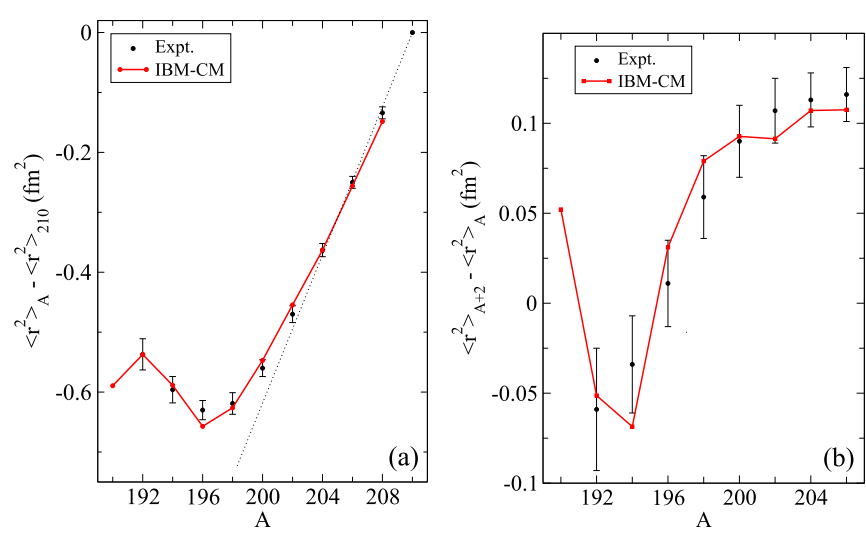

FIG. 14. (Color online) (a) Charge mean-square radii for the Po nuclei. (b) Isotopic shift for the Po nuclei. The data are taken from Ref. [118]

To calculate the isotope shifts, we have used the standard IBM-CM expression for the nuclear radius,

$$
\begin{aligned}
r^{2}= & r_{c}^{2}+\hat{P}_{N}^{\dagger}\left(\gamma_{N} \hat{N}+\beta_{N} \hat{n}_{d}\right) \hat{P}_{N} \\
& +\hat{P}_{N+2}^{\dagger}\left(\gamma_{N+2} \hat{N}+\beta_{N+2} \hat{n}_{d}\right) \hat{P}_{N+2} .
\end{aligned}
$$

The four parameters appearing in this expression are fitted to the experimental data, corresponding to the radii of $A=$ 192-198 even-even Po isotopes [118]. The resulting values are $\gamma_{N}=-0.108 \mathrm{fm}^{2}, \beta_{N}=0.152 \mathrm{fm}^{2}, \gamma_{N+2}=-0.022 \mathrm{fm}^{2}$, and $\beta_{N+2}=0.027 \mathrm{fm}^{2}$. Note that in determining these parameters we took as reference point ${ }^{204} \mathrm{Po}$ instead of ${ }^{210} \mathrm{Po}$, which is the reference for the experimental data.

Panel (a) of Fig. 14 shows the value of the radius relative to $A=210$. The first important fact is the strong deviation from the linear trend (marked with the dotted line) at $A=198$ and downwards, which is showing the onset of deformation confirmed in several recent experimental papers $[116,118]$. To see this fact more clearly, we also present the value of the isotopic shift which enhances the appearance of the deformation. We mention the very good quantitative agreement between the IBM-CM and the experimental data, which confirms that the interplay between the $[N]$ and the $[N+2]$ contributions in the $0^{+}$ground-state wave function along the whole chain of Po isotopes is well described by the model. Comparing with the data for the nearby $\mathrm{Hg}$ and $\mathrm{Pt}$ isotopes, one notices that the range of variation of the isotopic shift for $\mathrm{Hg}$ is only $\approx 0.02 \mathrm{fm}^{2}$, while for Pt this amounts to $\approx 0.1 \mathrm{fm}^{2}$ and, finally, for Po becomes $\approx 0.2 \mathrm{fm}^{2}$. This fact is telling us how abrupt the onset of deformation in the Po is. Note the strong similarity of Fig. 14(b) with the Pt case [58], where there is also an abrupt drop of the isotopic shift at $A=184-186$.

\section{QUADRUPOLE INVARIANTS AND NUCLEAR DEFORMATION}

The IBM can provide us with both the energy spectra and the corresponding wave functions, as well as all derived observables $[B(E 2)$ 's, quadrupole moments, radii, ...], working within the laboratory frame, as well as the corresponding mean-field energy surface, defining a nuclear shape over the 

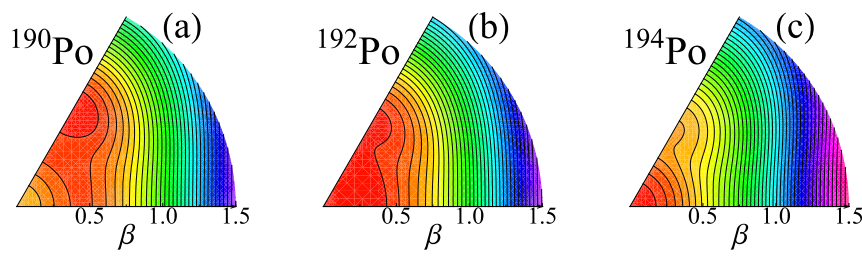

FIG. 15. (Color online) Matrix coherent-state calculation for ${ }^{190-194}$ Po, corresponding with the present IBM-CM Hamiltonian (Table II). The energy spacing between adjacent contour lines equals $100 \mathrm{keV}$ and the deepest energy minimum is set to zero, corresponding to the red color.

$\beta-\gamma$ intrinsic frame. This geometric interpretation of the IBM can be constructed using the intrinsic state formalism, as proposed in Refs. [161-165]. The extension of this formalism to describe simultaneously regular and intruder configurations was proposed by Frank et al., introducing a matrix coherentstate method [166-169]. In Refs. [59,64] a detailed description of the method and its application to $\mathrm{Pt}$ and $\mathrm{Hg}$ isotopes, respectively, can be found. In Figs. 15(a)-15(c) the IBM-CM mean-field energy surfaces are depicted for ${ }^{190} \mathrm{Po},{ }^{192} \mathrm{Po}$, and ${ }^{194} \mathrm{Po}$, respectively. In ${ }^{190} \mathrm{Po}$ the energy surface presents an oblate minimum, which evolves into a very flat surface for ${ }^{192} \mathrm{Po}$ and in a spherical shape for ${ }^{194} \mathrm{Po}$. Note that from Fig. 3 in Ref. [127], the ${ }^{192} \mathrm{Po}$ and ${ }^{194} \mathrm{Po}$ nuclei exhibit an oblate minimum; however, in reaching ${ }^{190} \mathrm{Po}$, the energy minimum switches over to become a prolate minimum, with a very similar deformation.

Even though the shape of the nucleus is not an experimental observable, it is still possible to extract from the data direct information about various moments characterizing the nuclear shape corresponding with a given eigenstate. Using Coulomb excitation, it is possible to extract the most important diagonal and nondiagonal quadrupole and octupole matrix elements, including their relative signs and, in a model-independent way, extract information about nuclear deformation, as shown by Kumar, Cline, and co-workers [170-174].

The essential point is the introduction of an "equivalent ellipsoid" view of a given nucleus [170] corresponding to a uniformly charged ellipsoid with the same charge, $\left\langle r^{2}\right\rangle$, and $E 2$ moments as the nucleus characterized by a specific eigenstate $[170,175]$.

From the theoretical point of view, the nuclear shape can be calculated using the quadrupole shape invariants. The quadrupole deformation corresponds to

$$
q_{2, i}=\sqrt{5}\left\langle 0_{i}^{+}\left|[\hat{Q} \times \hat{Q}]^{(0)}\right| 0_{i}^{+}\right\rangle .
$$

For the triaxial rigid rotor model [176] it is directly connected with the deformation parameter

$$
q_{2}=q^{2}
$$

where $q$ denotes the nuclear intrinsic quadrupole moment.

To calculate analytically the quadrupole shape invariants characterizing the nucleus in its ground-state and low-lying excited states, it is necessary to resort to a closure relation,
TABLE IV. Quadrupole shape invariants for the ${ }^{190-208}$ Po isotopes.

\begin{tabular}{lccccc}
\hline \hline Isotope & State & $q^{2}\left(e^{2} \mathrm{~b}^{2}\right)$ & Isotope & State & $q^{2}\left(e^{2} \mathrm{~b}^{2}\right)$ \\
\hline 190 & $0_{1}^{+}$ & $6.2^{\mathrm{a}}$ & 200 & $0_{1}^{+}$ & $1.1^{\mathrm{a}}$ \\
& $0_{2}^{+}$ & $5.7^{\mathrm{a}}$ & & $0_{2}^{+}$ & $0.9^{\mathrm{a}}$ \\
\multirow{3}{*}{192} & $0_{1}^{+}$ & $4.8^{\mathrm{a}}$ & 202 & $0_{1}^{+}$ & $1.1^{\mathrm{a}}$ \\
& $0_{2}^{+}$ & $4.5^{\mathrm{a}}$ & & $0_{2}^{+}$ & $0.9^{\mathrm{a}}$ \\
194 & $0_{1}^{+}$ & 3.6 & 204 & $0_{1}^{+}$ & $0.7^{\mathrm{a}}$ \\
& $0_{2}^{+}$ & 3.5 & & $0_{2}^{+}$ & $0.7^{\mathrm{a}}$ \\
& $0_{1}^{+}$ & 1.7 & 206 & $0_{1}^{+}$ & $0.6^{\mathrm{a}}$ \\
& $0_{2}^{+}$ & 2.8 & & $0_{2}^{+}$ & $0.4^{\mathrm{a}}$ \\
& $0_{1}^{+}$ & 1.4 & 208 & $0_{1}^{+}$ & $0.4^{\mathrm{a}}$ \\
& $0_{2}^{+}$ & 1.2 & & $0_{2}^{+}$ & $0.1^{\mathrm{a}}$
\end{tabular}

${ }^{\text {a The effective charges have been taken from neighboring isotopes }}$ (see Table II).

$\mathbf{1}=\sum_{J, i, M}\left|J_{i} M\right\rangle\left\langle J_{i} M\right|$, leading to the expression

$$
q_{2, i}=\sum_{r}\left\langle 0_{i}^{+}\|\hat{Q}\| 2_{r}^{+}\right\rangle\left\langle 2_{r}^{+}\|\hat{Q}\| 0_{i}^{+}\right\rangle
$$

In Table IV, we present the theoretical value of $q^{2}$ corresponding to the $0_{1}^{+}$and $0_{2}^{+}$states for the whole chain of Po isotopes. In this case, only very few experimental values of the reduced $E 2$ matrix elements $\left\langle 0_{i}^{+}\|\hat{Q}\| 2_{r}^{+}\right\rangle$have been measured [115] besides the strongest and dominating $\left\langle 0_{1}^{+}\|\hat{Q}\| 2_{1}^{+}\right\rangle$matrix element. Because of the limited number of reduced $E 2$ matrix elements, going into $r=2,3, \ldots$ final states, it is not possible to extract reliable experimental values. The reason that it is not possible to extract the triaxial shape variable $\delta$ is the same reason for not including this variable in the discussion. The main conclusion of this table is that the deformation of both $0_{1,2}^{+}$states is very similar and is dropping when moving from midshell, at $N=104$, to the end of the shell, at $N=126$ [115].

Starting from the quadrupole invariant (15), one can extract a value of the deformation $\beta$ (see, e.g., Refs. $[173,177,178]$ )

$$
\beta=\frac{4 \pi \sqrt{q^{2}}}{3 Z e r_{0}^{2} A^{2 / 3}},
$$

where $e$ is the proton charge and $r_{0}=1.2 \mathrm{fm}$. Thus, we can extract values for $\beta$ corresponding to the ground-state $0_{1}^{+}$and the first excited $0_{2}^{+}$state.

The resulting $\beta$ values, extracted from Eq. (18), are shown in Fig. 16(a), and one notices an overall decrease, albeit with a plateau in the mass region $194 \leqslant A \leqslant 202$, which is the region corresponding with an overall change in the energy scale for the first excited $2_{1}^{+}$state.

We also provide a different measure of the deformation value and extract a value of $\beta$ starting from a given $B(E 2)$ value through the expression

$$
\beta=\frac{4 \pi \sqrt{B(E 2 ; J \rightarrow J-2)}}{3 Z e r_{0}^{2} A^{2 / 3}\langle J 020 \mid J-20\rangle},
$$

where $\left\langle j_{1} m_{1} j_{2} m_{2} \mid j m\right\rangle$ is a Clebsch-Gordan coefficient. 


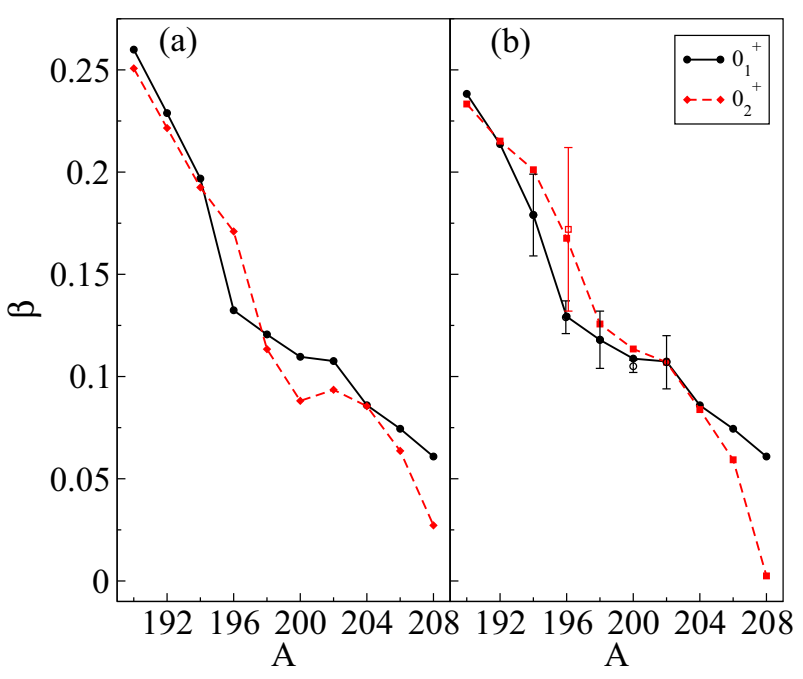

FIG. 16. (Color online) Comparison of the value of $\beta$ extracted from the theoretical quadrupole shape invariants (a) and the ones extracted from experimental and theoretical $B(E 2)$ values (b), corresponding to the $\mathrm{O}_{1}^{+}$and $\mathrm{O}_{2}^{+}$states.

In particular, we extract the value of $\beta$, derived from the $B\left(E 2 ; 2_{1}^{+} \rightarrow 0_{1}^{+}\right)$value, but also for any other $B\left(E 2 ; J^{+} \rightarrow\right.$ $(J-2)^{+}$) value along the yrast band. The extracted $\beta$ values then give interesting information about a possible variation along the band. Note that we extract the value for $0_{1}^{+}$from $B\left(E 2 ; 2_{1}^{+} \rightarrow 0_{1}^{+}\right)$and for $0_{2}^{+}$from $B\left(E 2 ; 6_{1}^{+} \rightarrow 4_{1}^{+}\right)$. The corresponding values are plotted in Fig. 16(b) and are fully consistent with the results, as shown in Fig. 16(a). They are slightly smaller because of using only a single but dominant $B\left(E 2 ; 2_{1}^{+} \rightarrow 0_{1}^{+}\right)$reduced transition probability.

The results for the Po nuclei are contrasting with the nearby $\mathrm{Hg}$ and $\mathrm{Pt}$ isotopes. In an analysis similar to the one carried out here, but for the $\mathrm{Hg}$ isotopes (see Figs. 2 and 3 in Ref. [179]), the $\beta$ values extracted from the IBM-CM results exhibit a strong difference in the interval $180 \leqslant A \leqslant 190$. This is consistent with the $\beta$ values extracted from the $B\left(E 2 ; 2_{1}^{+} \rightarrow\right.$ $0_{1}^{+}$) (showing the less deformed oblate structure) and from the $B\left(E 2 ; 6_{1}^{+} \rightarrow 4_{1}^{+}\right.$) (associated with a more deformed prolate structure) and quite different from the overall smooth drop in the $\beta$ values extracted in the Po isotopes. Comparing with the Pt nuclei, in which fewer data were available (see Fig. 3.27 in Ref. [5]), down to mass $A=188, \beta$ values extracted from the $B\left(E 2 ; 2_{1}^{+} \rightarrow 0_{1}^{+}\right)$and the $B\left(E 2 ; 6_{1}^{+} \rightarrow 4_{1}^{+}\right)$reduced $E 2$ transition probabilities do not show the same large separation as in the case of the $\mathrm{Hg}$ isotopes. In the $\mathrm{Pt}$ case, a rather smooth transition (a bump) from the region with $A \geqslant 188$ moving towards the midshell region exists. This was shown to be a consequence of the rather strong mixing between regular and intruder configurations [57,58]. The behavior of $\beta$, within the context of the IBM-CM, indicated a similar bump as a function of mass number [59].

Interesting information about the changing collective structure along the yrast band for the Po can be extracted studying the variation of the moment of inertia as one moves up the band structure. A quantitative measure that is often used is the

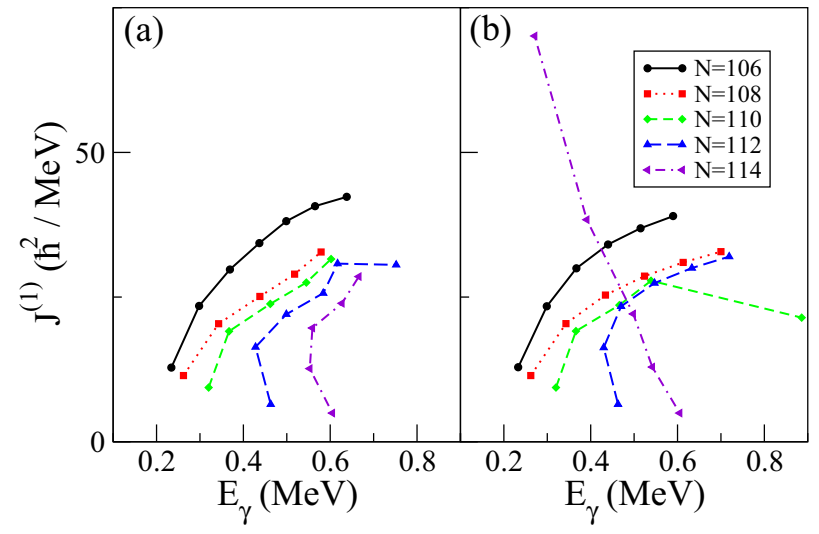

FIG. 17. (Color online) Comparison of the experimental (a) and theoretical (b) kinematic moment of inertia for selected Po isotopes with $106 \leqslant N \leqslant 114$.

kinematic moment of inertia $J^{(1)}$, which is defined as $[41,180]$

$$
J^{(1)}=\frac{\hbar^{2}}{2}\left\{\frac{d E}{d[J(J+1)]}\right\}^{-1} \approx \frac{\hbar^{2}(2 J-1)}{2 E_{\gamma}[J \rightarrow(J-2)]},
$$

where $E_{\gamma}[J \rightarrow(J-2)]$ is the energy difference $E(J)-$ $E(J-2)$. For an ideal rigid rotor, with a moment of inertia that is independent of the angular momentum $J$, the kinematic moment of inertia reduces to a constant.

In constructing the experimental $J^{(1)}$ values, we use the yrast band energies for the corresponding $J^{\pi}$ values. However, approaching the high-spin levels with $8^{+}, 10^{+}$, and $12^{+}$, it can happen that noncollective (mainly broken-pair configurations) do cross the collective structure, giving also rise to isomeric states as a fingerprint. Consequently, considering those states, the smooth variation of $J^{(1)}$ will change into a backbending pattern. Experimental data on lifetimes, $\gamma$-ray intensities characterizing the decay within a band structure, and the coincidence data with $\gamma$ rays from the low-energy transitions in the experimental yrast bands have been used to construct the resulting $J^{(1)}$ values. In particular, for the Po nuclei (see Refs. [8,85,89,90,111,114]), we cover the $106 \leqslant N \leqslant 114$ (190 $\leqslant A \leqslant 198$ ), region, with the results presented in Fig. 17. A similar analysis spanning a smaller set of Po nuclei was carried out in Refs. [8,86,110,111]. One observes a very smooth increase along the yrast band, moving up to rather high spin values in the $A=190$ (up to spin $14^{+}$), 192, and 194 (up to spin $10^{+}$) isotopes, consistent with the energy spectra as shown in Fig. 1. It is only for the higher-spin states, which are of a noncollective broken-pair nature, that very small energy differences appear, not at all consistent with collective excitations and giving rise to strong backbending (not shown here). From $A=198$ and onwards, a different energy pattern for $J^{(1)}$ is observed. For the lower part of the band, up to the $6_{1}^{+}$level, the values of $E_{\gamma}$ are roughly constant, indicating the typical energy differences of a vibrational structure. The fairly correct reproduction of this trend by the IBM-CM results indicates that the major physics content is rather well described.

It is also interesting to compare-making use of the experimental data on band structure and shape coexistence in the $\mathrm{Pb}$ region - the Po nuclei with the neighboring $\mathrm{Pb}, \mathrm{Hg}$, and $\mathrm{Pt}$ isotopes to appreciate similarities and differences, covering 


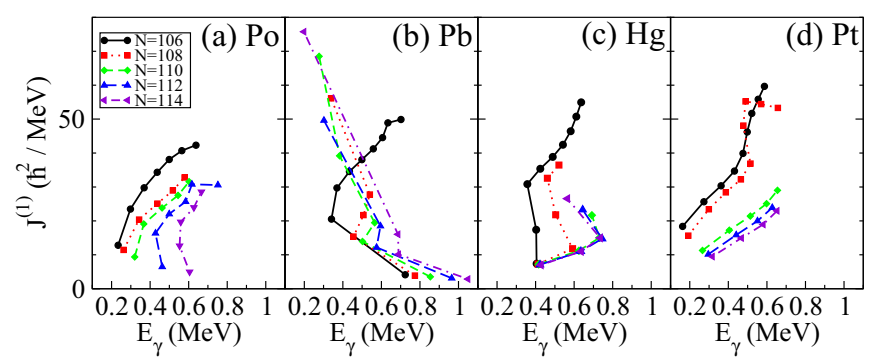

FIG. 18. (Color online) Comparison of the experimental moment of inertia in $\mathrm{Po}(\mathrm{a}), \mathrm{Pb}(\mathrm{b}), \mathrm{Hg}(\mathrm{c})$, and $\mathrm{Pt}$ (d) for selected isotones with $106 \leqslant N \leqslant 114$.

the same neutron interval $106 \leqslant N \leqslant 114$, as shown in Fig. 18 . The data used to construct the $J^{(1)}$ band for the $\mathrm{Po}, \mathrm{Pb}, \mathrm{Hg}$, and $\mathrm{Pt}$ isotopes have been taken from the references given in Sec. II and Ref. [181].

An obvious observation from Fig. 18 is the fact that at $N=106$, i.e., the nuclei ${ }^{190} \mathrm{Po},{ }^{188} \mathrm{~Pb},{ }^{186} \mathrm{Hg}$, and ${ }^{184} \mathrm{Pt}$, from $J^{\pi}=6^{+}$onwards, a very similar variation in the value of $J^{(1)}$ results. The starting point in these nuclei is $\approx 30 \hbar^{2} \mathrm{MeV}^{-1}$, followed by a similar slope up to spins $12^{+}$and $14^{+}$. In view of the well-established prolate character in the case of ${ }^{188} \mathrm{~Pb}$ and ${ }^{186} \mathrm{Hg}$, it is a convincing argument that the ground-state band in both ${ }^{190} \mathrm{Po}$ and ${ }^{184} \mathrm{Pt}$, from spins $4^{+}, 6^{+}$, and onwards, behave like a prolate band, too. One also notices that because the ground state $0^{+}$and first excited $2^{+}$state in ${ }^{188} \mathrm{~Pb}$ retain mainly a spherical character, the low $E_{\gamma}$ part exhibits a different sloping start, very much like it is the case in ${ }^{186} \mathrm{Hg}$. In the latter nucleus, there appears an offset of the prolate band versus the ground-state less-deformed band (oblate, anharmonic vibrational) causing almost constant values of $E_{\gamma}$ to result for the $2^{+}$and $4^{+}$states. In ${ }^{190} \mathrm{Po}$ and ${ }^{184} \mathrm{Pt}$, a very smooth behavior results along the yrast band, extending up to rather high-spin states and this for the whole region $106 \leqslant N \leqslant 114$. One also observes a considerable drop in the value of $J^{(1)}$ in this interval, which is of the order of $\approx 10 \hbar^{2} \mathrm{MeV}^{-1}$. In this respect, the Po and Pt nuclei exhibit a rather similar collective behavior, with the "intruder" states being not that obvious from the experimental data on excitation energies nor the corresponding $B(E 2)$ electromagnetic properties. Only radii and $\alpha$-decay hindrance factors point towards the need to consider both configuration (regular $[N]$ and intruder $[N+2]$ ) spaces.

\section{CONCLUSIONS AND OUTLOOK}

The Po region constitutes one of the clearer examples where shape coexistence plays a key role in explaining the major observed features such as the evolving energy spectra, with mass number decreasing from the neutron closed shell at $N=126$, approaching the midshell region at $N=104$, the electromagnetic properties, in particular the $B(E 2)$ systematics, nuclear mean-square charge radii, $\alpha$-decay hindrance factors, etc. These isotopes are situated in the region of the $\mathrm{Pb}$, $\mathrm{Hg}$, and $\mathrm{Pt}$ isotopes where the proximity of the $Z=82$ proton closed shell, and its stabilizing effect to keep nuclei mainly into a spherical shape, in particular for the $\mathrm{Pb}$ nuclei, is very evident. These series of isotopes can be divided in two groups. On the one hand, there are the $\mathrm{Pb}$ and $\mathrm{Hg}$ nuclei, in which two (or even three for the $\mathrm{Pb}$ nuclei) types of configurations coexist and are experimentally well documented, characterized by energy spectra that exhibit the characteristic parabolic behavior of the intruder band structure, minimizing its excitation energy at the midshell, $N=104$ [52,59]. On the other hand, there are the Pt and Po isotopes for which one cannot disentangle easily the presence of two distinct band structures. Still, in both the Pt and the Po isotopes, approaching the midshell point, the first excited $0^{+}$state behaves in an unexpected way, dropping seriously in excitation energy which can be used as an indirect hint for the presence of shape coexistence, however, in a concealed way [58].

In the present paper, we have carried out an extensive study of the chain of isotopes ${ }^{190-208}$ Po using the IBM, including pair boson excitations across the $Z=82$ proton closed shell (called the intruder configuration space), and their interaction with the regular configuration space, called the IBM-CM. We have determined the Hamiltonian and the E2 operator describing this interacting system of bosons through a least-squares fit to the known experimental data. This then results in the energy spectra, and, moreover, the calculation of many different observables such as the $B(E 2)$ values, nuclear mean-square charge radii, gyromagnetic factors, and $\alpha$-decay-hindrance factors, giving the possibility to test the nuclear dynamics. In particular, the latter three observables are shown to serve as fingerprints to test the relative composition of the nuclear wave function into its "regular" and "intruder" components, and thus are strong indicators for the presence of shape coexisting structures.

A very important issue concerns deriving information on the nuclear deformation properties. In particular, recent Coulomb excitation experiments on nuclei far from stability made it possible to extract a set of reduced E2 matrix elements $\left\langle 0_{i}^{+}\|\hat{T}(E 2)\| 2_{f}^{+}\right\rangle$. These matrix elements can be used to construct the so-called "quadrupole shape invariants" for the $0_{1}^{+}$and $0_{2}^{+}$states. We have given particular attention to how $\beta$ values can be derived from these invariants, or, alternatively, from known experimental $B(E 2)$ values, and compared both approaches in the case of the Po nuclei. Thereby, a clear picture of the shape coexistence phenomenon in Po arises. There are two families of configurations, one slightly deformed (rather $\gamma$-unstable) or spherical that corresponds to the regular configurations, while the other more deformed and corresponding to the intruder configurations. Because of the lack of experimental information it is not possible to determine unambiguously the shape connected to the two families of configurations and, indeed, through the use of mean-field results (see Sec. III) we assume an oblate shape for the intruder states, although the moment of inertia seems to suggest a prolate shape for the ground state of ${ }^{190} \mathrm{Po}$. Both configurations have a rather large interplay and in the midshell region with the two $0^{+}$unperturbed bandheads being degenerate in energy, implying important mixing between the two configuration spaces. We have found that, in contrast with the $\mathrm{Hg}$ nuclei, the difference in the quadrupole deformation extracted for the $0_{1}^{+}$and $\mathrm{O}_{2}^{+}$states is quite small and the mass dependence very similar, independent of the method used to extract the quadrupole deformation. 
Concluding, it looks like the data available at present, based on our present study of the Po isotopes and a comparison with the Pt isotopes, points towards rather similar structures. In both cases the ground-state at the midshell is composed mainly from the intruder configurations, although in the Po isotopes, only the second part of the neutron shell $N=82-126$ has been studied experimentally. For the Pt isotopes, almost the whole shell has been covered, but for the Po nuclei, because of the sparse character of the known data in and below $N=106$, $A=190$, a detailed comparison is very difficult. Moreover, the similar character of the Po and Pt isotopes is supported from a comparison of the kinematic moments of inertia for the Po and Pt isotopes: Both exhibit a smooth variation as a function of neutron number in the interval $106 \leqslant N \leqslant 114$. Comparing with the corresponding moments of inertia in the $\mathrm{Pb}$ and $\mathrm{Hg}$ isotopes, the nuclei ${ }^{190} \mathrm{Po},{ }^{188} \mathrm{~Pb},{ }^{186} \mathrm{Hg}$, and ${ }^{184} \mathrm{Pt}$ (starting at spin $6^{+}$) are very similar, resulting in the suggestion that from that spin, and onwards, the ${ }^{190} \mathrm{Po}$ ground band behaves like a prolate band. This is further corroborated by a comparison of the known experimental excitation energies for the prolate bands that have been observed in ${ }^{186} \mathrm{Hg}$ and in ${ }^{188} \mathrm{~Pb}$ for states with spin from $6^{+}$to $10^{+}$. They match very well the corresponding energies in the yrast band structure in
${ }^{190}$ Po. Possibilities to test this proposal might come partly from multiple Coulomb excitation with higher-energy projectile ions (HIE-ISOLDE) and, possibly at a later stage, using oneand two-nucleon transfer reactions and studying $E 0$ properties for the Po nuclei, which may lead to the direct observation of a low-lying excited $0^{+}$state, as suggested by the present study.

\section{ACKNOWLEDGMENTS}

We are very grateful to $\mathrm{N}$. Kesteloot for generous sharing of their most recent results on Coulomb excitation $(A=196-$ 198). We thank M. Huyse and P. Van Duppen for continuous interest in this research topic and J. L. Wood for stimulating discussions in various stages of this work. Financial support from the "FWO-Vlaanderen" (K.H. and J.E.G.R.) and the InterUniversity Attraction Poles Programme-Belgian StateFederal Office for Scientific, Technical and Cultural Affairs (IAP Grant No. P7/12) is acknowledged. This work has also been partially supported by the Spanish Ministerio de Economía y Competitividad and the European regional development fund (FEDER) under Project No. FIS2011-28738C02-02 and by Spanish Consolider-Ingenio 2010 (Grant No. CPANCSD2007-00042).
[1] H. Morinaga, Phys. Rev. 101, 254 (1956).

[2] P. Van Duppen, E. Coenen, K. Deneffe, M. Huyse, K. Heyde, and P. Van Isacker, Phys. Rev. Lett. 52, 1974 (1984).

[3] A. N. Andreyev et al., Nature (London) 405, 430 (2000).

[4] K. Heyde, P. Van Isacker, M. Waroquier, J. L. Wood, and R. A. Meyer, Phys. Rep. 102, 291 (1983).

[5] J. L. Wood, K. Heyde, W. Nazarewicz, M. Huyse, and P. Van Duppen, Phys. Rep. 215, 101 (1992).

[6] K. Heyde and J. L. Wood, Rev. Mod. Phys. 83, 1467 (2011).

[7] Y. Blumenfeld, T. Nilsson, and P. Van Duppen, Nobel Symposium 152: Physics with Radioactive Beams, edited by C. Fahlander and B. Jonson [Phys. Scr. T 152, 014023 (2013)].

[8] R. Julin, K. Helariutta, and M. Muikku, J. Phys. G 27, R109 (2001).

[9] A. Gade and Th. Glasmachter, Prog. Part. Nucl. Phys. 60, 161 (2008).

[10] A. Görgen, J. Phys. 37, 103101 (2010).

[11] P. G. Hansen and J. A. Tostevin, Annu. Rev. Nucl. Part. Sci. 53, 219 (2003).

[12] M. Pfützner, M. Karny, L. V. Grgigorenko, and K. Riisager, Rev. Mod. Phys. 84, 567 (2012).

[13] T. Baumann, A. Spyrou, and M. Thoennessen, Rep. Progr. Phys. 75, 036301 (2012).

[14] K. Blaum, J. Dilling, and W. Nörtershäuser, Nobel Symposium 152: Physics with Radioactive Beams, edited by C. Fahlander and B. Jonson [Phys. Scr. T 152, 014017 (2013)].

[15] B. Cheal and K. T. Flanagan, J. Phys. 37, 113101 (2010).

[16] G. Neyens, Rep. Progr. Phys. 66, 633 (2003).

[17] J. L. Wood, E. F. Zganjar, C. De Coster, and K. Heyde, Nucl. Phys. A 651, 323 (1999).

[18] T. Kibédi and R. H. Spear, At. Data Nucl. Data Tables 89, 77 (2005).

[19] E. Caurier and F. Nowacki, Acta Phys. Pol. B 30, 705 (1999).

[20] B. A. Brown and W. D. M. Rae, Nucl. Data Sheets 120, 115 (2014).
[21] N. Shimizu, T. Abe, Y. Tsunoda, Y. Utsuno, T. Yoshida, T. Mizusaki, M. Honma, and T. Otsukaa, Prog. Theor. Exp. Phys. 2012, 01A205 (2012).

[22] E. Caurier, G. Martínez-Pinedo, F. Nowacki, A. Poves, and A. P. Zuker, Rev. Mod. Phys. 77, 427 (2005).

[23] R. Bengtsson, T. Bengtsson, J. Dudek, G. Leander, W. Nazarewicz, and Jing-Ye Zhang, Phys. Lett. B 183, 1 (1987).

[24] R. Bengtsson and W. Nazarewicz, Z. Phys. A 334, 269 (1989).

[25] W. Nazarewicz, Phys. Lett. B 305, 195 (1993).

[26] M. Bender, P.-H. Heenen, and P.-G. Reinhard, Rev. Mod. Phys. 75, 121 (2003).

[27] T. H. R. Skyrme, Nucl. Phys. 9, 615 (1959).

[28] D. Vautherin and D. M. Brink, Phys. Rev. C 5, 626 (1972).

[29] J. Erler, P. Klüpfel, and P.-G. Reinhard, J. Phys. 38, 033101 (2011).

[30] D. Gogny, in Proceedings of the International Conference on Nuclear Physics, Münich, 1973, edited by J. De Boer and H. J. Mang (North Holland, Amsterdam, 1973).

[31] D. Gogny, in Proceedings of the International Conference on Nuclear Selfconsistent Fields, 1975, Trieste, edited by G. Ripka and M. Porneuf (North-Holland, Amsterdam, 1975).

[32] J. Dechargé and D. Gogny, Phys. Rev. C 21, 1568 (1980).

[33] M. Girod, P. Dessagne, M. Bernas, M. Langevin, F. Pougheon, and P. Roussel, Phys. Rev. C 37, 2600 (1988).

[34] J. Libert, M. Girod, and J.-P. Delaroche, Phys. Rev. C 60, 054301 (1999).

[35] J. D. Walecka, Ann. Phys. (NY) 83, 491 (1974).

[36] B. D. Serot, Rep. Prog. Phys. 55, 1855 (1992).

[37] P.-G. Reinhard, Rep. Prog. Phys. 52, 439 (1989).

[38] B. D. Serot and J. D. Walecka, Advances in Nuclear Physics (Plenum Press, New York, 1986), Vol. 16, p. 1.

[39] P. Ring, Progr. Part. Nucl. Phys. 37, 193 (1996).

[40] T. Nikšić, D. Vretenar, and P. Ring, Progr. Part. Nucl. Phys. 66, 519 (2011). 
[41] A. Bohr and B. R. Mottelson, Nuclear Structure (W. A Benjamin, Reading MA, 1975), Vol. II.

[42] D. J. Rowe and J. L. Wood, Fundamentals of Nuclear Models. Foundational Models (World Scientific, Singapore, 2010).

[43] L. Próchniak and S. G. Rohozinski, J. Phys. 36, 123101 (2009).

[44] J. P. Delaroche, M. Girod, J. Libert, H. Goutte, S. Hilaire, S. Péru, N. Pillet, and G. F. Bertsch, Phys. Rev. C 81, 014303 (2010).

[45] M. Matsuyanagi, M. Matsuo, T. Nakatsukasa, N. Hinohara, and K. Sato, J. Phys. 37, 064018 (2010).

[46] F. Iachello and A. Arima, The Interacting Boson Model (Cambridge University Press, Cambridge, UK, 1987).

[47] P. D. Duval and B. R. Barrett, Phys. Lett. B 100, 223 (1981).

[48] P. D. Duval and B. R. Barrett, Nucl. Phys. A 376, 213 (1982).

[49] K. Heyde, J. Jolie, J. Moreau, J. Ryckebusch, M. Waroquier, P. Van Duppen, M. Huyse, and J. L. Wood, Nucl. Phys. A 466, 189 (1987).

[50] K. Heyde, J. Schietse, and C. De Coster, Phys. Rev. C 44, 2216 (1991).

[51] J. Pakarinen et al., Phys. Rev. C 75, 014302 (2007).

[52] R. Fossion, K. Heyde, G. Thiamova, and P. Van Isacker, Phys. Rev. C 67, 024306 (2003).

[53] V. Hellemans, R. Fossion, S. D. Baerdemacker, and K. Heyde, Phys. Rev. C 71, 034308 (2005).

[54] V. Hellemans, S. De Baerdemacker, and K. Heyde, Phys. Rev. C 77, 064324 (2008).

[55] M. K. Harder, K. T. Tang, and P. Van Isacker, Phys. Lett. B 405, 25 (1997).

[56] S. L. King et al., Phys. Lett. B 433, 82 (1998).

[57] J. E. García-Ramos and K. Heyde, Nucl. Phys. A 825, 39 (2009).

[58] J. E. García-Ramos, V. Hellemans, and K. Heyde, Phys. Rev. C 84, 014331 (2011).

[59] J. E. García-Ramos, K. Heyde, L. M. Robledo, and R. Rodriguez-Guzmán, Phys. Rev. C 89, 034313 (2014).

[60] E. A. McCutchan and N. V. Zamfir, Phys. Rev. C 71, 054306 (2005), and private communication.

[61] E. A. McCutchan, R. F. Casten, and N. V. Zamfir, Phys. Rev. C 71, 061301(R) (2005).

[62] A. Barfield, B. R. Barrett, K. A. Sage, and P. D. Duval, Z. Phys. A 311, 205 (1983).

[63] A. Barfield and B. R. Barrett, Phys. Lett. B 149, 277 (1984).

[64] J. E. García-Ramos and K. Heyde, Phys. Rev. C 89, 014306 (2014).

[65] K. Nomura, N. Shimizu, and T. Otsuka, Phys. Rev. Lett. 101, 142501 (2008).

[66] K. Nomura, N. Shimizu, and T. Otsuka, Phys. Rev. C 81, 044307 (2010).

[67] A. Arima, T. Otsuka, F. Iachello, and I. Talmi, Phys. Lett. B 66, 205 (1977).

[68] T. Otsuka, A. Arima, F. Iachello, and I. Talmi, Phys. Lett. B 76, 139 (1978).

[69] K. Nomura, T. Otsuka, R. Rodríguez-Guzmán, L. M. Robledo, and P. Sarriguren, Phys. Rev. C 83, 014309 (2011).

[70] K. Nomura, T. Nikšić, T. Otsuka, N. Shimizu, and D. Vretenar, Phys. Rev. C 84, 014302 (2011).

[71] K. Nomura, R. Rodríguez-Guzmán, and L. M. Robledo, Phys. Rev. C 87, 064313 (2013).

[72] K. Nomura, R. Rodríguez-Guzmán, L. M. Robledo, and N. Shimizu, Phys. Rev. C 86, 034322 (2012).

[73] B. Singh, Nucl. Data Sheets 99, 275 (2003).
[74] C. M. Baglin, Nucl. Data Sheets 113, 1871 (2012).

[75] B. Singh, Nucl. Data Sheets 107, 1531 (2006).

[76] H. Xiaolong, Nucl. Data Sheets 108, 1093 (2007).

[77] Z. Chunmei, Nucl. Data Sheets 95, 59 (2002).

[78] F. G. Kondev and S. Lalkovski, Nucl. Data Sheets 108, 1471 (2007).

[79] S. Zhu and F. G. Kondev, Nucl. Data Sheets 109, 699 (2008).

[80] C. J. Chiara and F. G. Kondev, Nucl. Data Sheets 111, 141 (2010).

[81] F. G. Kondev, Nucl. Data Sheets 109, 1527 (2008).

[82] M. J. Martin, Nucl. Data Sheets 108, 1583 (2007).

[83] M. S. Basania, Nucl. Data Sheets 121, 561 (2014).

[84] N. Fotiades et al., Phys. Rev. C 55, 1724 (1997).

[85] K. Helariutta et al., Phys. Rev. C 54, R2799 (1996).

[86] K. Helariutta et al., Eur. Phys. J. A 6, 289 (1999).

[87] W. Younes et al., Phys. Rev. C 52, R1723 (1995).

[88] D. Alber, R. Alfier, C. E. Bach, D. B. Fossan, H. Grawe, H. Kluge, M. Lach, K. H. Maier, M. Schramm, R. Schubart, M. P. Waring, L. Wood, H. Hübel, and Jing-ye Zhang, Z. Phys. A 339, 225 (1991).

[89] L. A. Bernstein et al., Phys. Rev. C 52, 621 (1995).

[90] M. Lach et al., Z. Phys. A 350, 207 (1994).

[91] A. Maj, H. Grawe, H. Kluge, A. Kuhnert, K. H. Mayer, J. Recht, N. Roy, H. Hübel, and M. Guttormsen, Nucl. Phys. A 509, 413 (1990).

[92] N. Bijnens et al., Phys. Rev. Lett. 75, 4571 (1995).

[93] N. Bijnens et al., Phys. Rev. C 58, 754 (1998).

[94] J. Wauters, P. Dendooven, M. Huyse, G. Reusen, P. Lievens, and P. Van Duppen (ISOLDE Collaboration), Z. Phys. A 344, 29 (1992).

[95] J. Wauters, P. Dendooven, M. Huyse, G. Reusen, P. Van Duppen, and P. Lievens, Phys. Rev. C 47, 1447 (1993).

[96] J. Wauters, N. Bijnens, P. Dendooven, M. Huyse, Han Yull Hwang, G. Reusen, J. von Schwarzenberg, P. Van Duppen, R. Kirchner, and E. Roeckl (ISOLDE Collaboration), Phys. Rev. Lett. 72, 1329 (1994).

[97] J. Wauters, N. Bijnens, H. Folger, M. Huyse, Han Yull Hwang, R. Kirchner, J. von Schwarzenberg, and P. Van Duppen, Phys. Rev. C 50, 2768 (1994).

[98] A. N. Andreyev et al., Phys. Rev. Lett. 82, 1819 (1999).

[99] N. Bijnens et al., Z. Phys. A 356, 3 (1996).

[100] R. G. Allatt et al., Phys. Lett. B 437, 29 (1998).

[101] A. N. Andreyev et al., J. Phys. G 25, 835 (1999).

[102] A. N. Andreyev et al., Phys. Rev. C 66, 014313 (2002).

[103] A. N. Andreyev et al., Phys. Rev. C 73, 044324 (2006).

[104] K. Van de Vel et al., Phys. Rev. C 68, 054311 (2003).

[105] K. Van de Vel et al., Eur. Phys. J. A 24, 57 (2005).

[106] P. Van Duppen and M. Huyse, Hyperfine Interact. 129, 149 (2000).

[107] M. Huyse, A. Andreyev, K. Van De Vel, P. Van Duppen, and R. Wyss, Hyperfine Interact. 132, 141 (2001).

[108] D. S. Delion, R. J. Liotta, C. Qi, and R. Wyss, Phys. Rev. C 90, 061303(R) (2014).

[109] D. S. Delion and R. J. Liotta, Phys. Rev. C 87, 041302(R) (2013).

[110] K. Van de Vel et al., Eur. Phys. J. A 17, 167 (2003).

[111] D. R. Wiseman et al., Eur. Phys. J. A 34, 275 (2007).

[112] T. Grahn et al., Phys. Rev. Lett. 97, 062501 (2006).

[113] T. Grahn et al., Nucl. Phys. A 801, 83 (2008).

[114] T. Grahn et al., Phys. Rev. C 80, 014324 (2009).

[115] N. Kesteloot et al. (unpublished). 
[116] M. D. Seliverstov et al., Phys. Lett. B 719, 362 (2013).

[117] M. D. Seliverstov et al., Phys. Rev. C 89, 034323 (2014).

[118] T. E. Cocolios et al., Phys. Rev. Lett. 106, 052503 (2011).

[119] A. de Shalit and I. Talmi, Nuclear Shell Theory (Academic Press, New York, 1963).

[120] I. Talmi, Simple Models of Complex Nuclei (Harwood Academic, Switzerland, 1993).

[121] J. J. Ressler et al., Phys. Rev. C 69, 034317 (2004).

[122] W. Younes and J. A. Cizewski, Phys. Rev. C 55, 1218 (1997).

[123] J. A. Cizewski and W. Younes, Z. Phys. A 358, 133 (1997).

[124] A. M. Oros, K. Heyde, C. De Coster, B. Decroix, R. Wyss, B. R. Barrett, and P. Navratil, Nucl. Phys. A 465, 107 (1999).

[125] F. R. May, V. V. Pashkevich, and S. Frauendorf, Phys. Lett. B 68, 113 (1977).

[126] N. A. Smirnova, P.-H. Heenen, and G. Neyens, Phys. Lett. B 569, 151 (2003).

[127] J. M. Yao, M. Bender, and P.-H. Heenen, Phys. Rev. C 87, 034322 (2013).

[128] Yue Shi, F. R. Xu, H. L. Liu, and P. M. Walker, Phys. Rev. C 82, 044314 (2010).

[129] Yue Shi, F. R. Xu, P. M. Walker, and G. D. Dracoulis, Phys. Rev. C 85, 064304 (2012).

[130] K. Heyde, C. De Coster, J. Jolie, and J. L. Wood, Phys. Rev. C 46, 541 (1992).

[131] K. Heyde, P. Van Isacker, and J. L. Wood, Phys. Rev. C 49, 559 (1994).

[132] C. De Coster, B. Decroix, K. Heyde, J. Jolie, H. Lehmann, and J. L. Wood, Nucl. Phys. A 651, 31 (1999).

[133] B. R. Barrett, G. D. Dracoulis, and R. A. Bark, Phys. Rev. C 43, R926 (1991).

[134] M. Scheck et al., Phys. Rev. C 83, 037303 (2011).

[135] J. Elseviers et al., Phys. Rev. C 84, 034307 (2011).

[136] R. D. Page et al., Phys. Rev. C 84, 034308 (2011).

[137] N. Bree et al., Phys. Rev. Lett. 112, 162701 (2014).

[138] L. Gaffney et al., Phys. Rev. C 89, 024307 (2014).

[139] K. Wrzosek-Lipska et al. (unpublished).

[140] E. Rapisarda (unpublished) and (private communication).

[141] D. D. Warner and R. F. Casten, Phys. Rev. C 28, 1798 (1983).

[142] P. O. Lipas, P. Toivonen, and D. D. Warner, Phys. Lett. B 155, 295 (1985).

[143] K. Heyde, P. Van Isacker, R. F. Casten, and J. L. Wood, Phys. Lett. B 155, 303 (1985).

[144] F. James, Minuit: Function Minimization and Error Analysis Reference Manual, Version 94.1 (CERN, Meyrin, Switzerland, 1994).

[145] N. J. Stone, At. Data Nucl. Data Tables 90, 75 (2005).

[146] N. J. Stone, Hyperfine Interact. 230, 7 (2015).

[147] G. Neyens, S. Ternier, N. Coulier, K. Vyvey, R. Coussement, and D. L. Balabanski, Nucl. Phys. A 625, 668 (1997).

[148] P. Van Duppen, M. Huyse, and J. L. Wood, J. Phys. G 16, 441 (1990).

[149] G. D. Dracoulis, A. E. Stuchbery, A. O. Machiavelli, C. W. Beausang, J. Burde, M. A. Deleplanque, R. M. Diamond, and F. S. Stephens, Phys. Lett. B 208, 365 (1988).

[150] G. D. Dracoulis, Phys. Rev. C 49, 3324 (1994).

[151] R. D. Page et al., in Proceedings of the 3rd International Conference on Exotic Nuclei and Atomic Masses ENAM, Hämeenlinna, Finland, July 2001, edited by J. Äystö, P. Dendooven, A. Jokinen, and M. Leino (Springer-Verlag, Berlin, Heidelberg, 2003), p. 309.
[152] G. D. Dracoulis, G. J. Lane, A. P. Byrne, T. Kibedi, A. M. Baxter, A. O. Macchiavelli, P. Fallon, and R. M. Clark, Phys. Rev. C 69, 054318 (2004).

[153] W. Satula and R. Wyss, Phys. Scr. T 56, 159 (1995).

[154] D. Karlgren, R. J. Liotta, R. Wyss, M. Huyse, K. Van de Vel, and P. Van Duppen, Phys. Rev. C 73, 064304 (2006).

[155] D. S. Delion, A. Florescu, M. Huyse, J. Wauters, P. Van Duppen, A. Insolia, and R. J. Liotta (ISOLDE Collaboration), Phys. Rev. Lett. 74, 3939 (1995).

[156] D. S. Delion, A. Florescu, M. Huyse, J. Wauters, P. Van Duppen, A. Insola, and R. J. Liotta (ISOLDE Collaboration), Phys. Rev. C 54, 1169 (1996).

[157] Chang Xu and Zhongzhou Ren, Phys. Rev. C 75, 044301 (2007).

[158] G. Ulm et al., Z. Phys. A 325, 247 (1986).

[159] E. Otten, in Nuclear Radii and Moments of Unstable Isotopes, Treatise on Heavy-Ion Science, Vol. 8, Nuclei Far from Stability, edited by D. A. Bromley (Plenum, New York, London, 1989), p. 517.

[160] H.-J. Kluge and W. Nötershäuser, Spectrochim. Acta, Part B: At. Spectrosc. 58, 1031 (2003).

[161] A. Bohr and B. R. Mottelson, Phys. Scr. 22, 468 (1980).

[162] J. N. Ginocchio and M. W. Kirson, Nucl. Phys. A 350, 31 (1980).

[163] A. E. L. Dieperink and O. Scholten, Nucl. Phys. A 346, 125 (1980).

[164] A. E. L. Dieperink, O. Scholten, and F. Iachello, Phys. Rev. Lett. 44, 1747 (1980).

[165] R. Gilmore, Lie Groups, Lie Algebras and Some of Their Applications (Wiley, New York, 1974).

[166] A. Frank, O. Castaños, P. Van Isacker, and E. Padilla, in Mapping the Triangle, AIP Conf. Proc. No. 638, edited by A. Aprahamian, J. A. Cizewski, S. Pittel, and N. V. Zamfir (AIP, Melville, NY, 2002), p. 23.

[167] A. Frank, P. Van Isacker, and C. E. Vargas, Phys. Rev. C 69, 034323 (2004).

[168] A. Frank, P. Van Isacker, and F. Iachello, Phys. Rev. C 73, 061302(R) (2006).

[169] I. O. Morales, A. Frank, C. E. Vargas, and P. Van Isacker, Phys. Rev. C 78, 024303 (2008).

[170] K. Kumar, Phys. Rev. Lett. 28, 249 (1972).

[171] D. Cline, Annu. Rev. Nucl. Part. Sci. 36, 683 (1986).

[172] C. Y. Wu, D. Cline, T. Czosnyka, A. Backlin, C. Baktash, R. M. Diamond, G. D. Dracoulis, L. Hasselgren, H. Kluge, B. Kotlinski, J. R. Leigh, J. O. Newton, W. R. Philips, S. H. Sie, J. Srebrny, and F. S. Stephens, Nucl. Phys. A 607, 178 (1996).

[173] E. Clément et al., Phys. Rev. C 75, 054313 (2007).

[174] J. Srebrny and D. Cline, Int. J. Mod. Phys. E 20, 422 (2011).

[175] J. L. Wood, Lecture Notes “Nuclear Collectivity” (University of York, York, UK, 2007).

[176] J. L. Wood, A.-M. Oros-Peusquens, R. Zaballa, J. M. Allmond, and W. D. Kulp, Phys. Rev. C 70, 024308 (2004).

[177] J. Srebrny et al., Nucl. Phys. A 766, 25 (2006).

[178] K. Wrzosek-Lipska et al., Phys. Rev. C 86, 064305 (2012).

[179] J. E. García-Ramos and K. Heyde, EPJ Web Conf. 93, 01004 (2015).

[180] S. G. Nilsson and I. Ragnarsson, Shapes and Shells in Nuclear Structure (Cambridge University Press, Cambridge, UK, 1995).

[181] G. D. Dracoulis, A. P. Byrne, and A. M. Baxter, Phys. Lett. B 432, 37 (1998). 